Peer Reviewed Paper openaccess Special Issue on Spectral Imaging in Synchrotron Light Facilities

\title{
New insights in osteocyte imaging by synchrotron radiation
}

\author{
H. Portier, ${ }^{a, b}$ C. Jaffré, ${ }^{a, c}$ C.M. Kewish, ${ }^{\text {d,e }}$ C. Chappard ${ }^{a}$ and S. Pallu ${ }^{a, b, *}$ \\ aUniversité de Paris, CNRS, Inserm, B3OA, F-75010 Paris, France \\ bUniversité d'Orléans, COST, 45100 Orléans, France. E-mail: stephane.pallu@univ-orleans.fr \\ 'Faculté des Sciences du Sport et Laboratoire Adaptations Physiologiques à l'Exercice et Réadaptation à l'effort (APERE)-EA3300, Allée Pascal \\ Grousset, 80025 Amiens Cedex 1, France
}

${ }^{d}$ Australian Nuclear Science and Technology Organisation (ANSTO), Australian Synchrotron, Clayton, VIC 3800, Australia

eDepartment of Chemistry and Physics, La Trobe University, Bundoora, VIC 3086, Australia

ORCID iDs

H. Portier: https://orcid.org/0000-0003-3328-3257

C. Chappard: https://orcid.org/0000-0001-9088-1582

C. Kewish: https://orcid.org/0000-0001-6242-7059

Bone health depends on the bone mineral density and mechanical strength, characterised quantitatively and inferred through qualitative parameters such as the trabecular and cortical micro-architecture, and other parameters describing the bone cells. Among these cells, the osteocyte has been recognised as the orchestrator of bone remodelling, playing a key role in directing osteoblastic and osteoclastic activities. Conventional optical and electron microscopies have greatly improved our understanding of the cell physiology mechanisms involved in different osteoarticular pathophysiological contexts, especially osteoporosis. More recently, imaging methods exploiting synchrotron radiation, such as $\mathrm{X}$-ray tomography, ptychography, and deep ultraviolet and Fourier transform infrared spectroscopies, have revealed new biochemical, chemical and 3D morphological information about the osteocyte lacuna, the surrounding matrix and the lacuna-canalicular network at spatial length scales spanning microns to tens of nanometres. Here, we review recent results in osteocyte lacuna and lacuna-canalicular network characterisation by synchrotron radiation imaging in human and animal models, contributing to new insights in different physiologic and pathophysiological situations.

Keywords: osteocyte imaging, synchrotron radiation, spectroscopy, tomography

\section{Introduction}

During the last decade, since the physiological role of the osteocyte has been known, osteocytes and their lacunacanalicular network (LCN) have been characterised by synchrotron radiation facilities. Here, we report at first, the involvement of this cell in bone physiology. Second, we present conventional ex vivo microscopic imaging methods devoted to the osteocyte. Third, we present the advantages of using synchrotron spectroscopy to optimise the characterisation of this cell. Last, we present and discuss the results obtained thanks to these different
Correspondence

S. Pallu (stephane.pallu@univ-orleans.fr)

Received: 19 February 2019

Revised: 12 December 2019

Accepted: 18 December 2019

Publication: 16 January 2020

doi: $10.1255 /$ jsi.2020.a3

ISSN: 2040-4565

\begin{abstract}
Citation
H. Portier, C. Jaffré, C.M. Kewish, C. Chappard and S. Pallu, "New

insights in osteocyte imaging by synchrotron radiation", J. Spectral Imaging

9, a3 (2020). https://doi.org/10.1255/jsi.2020.a3

(c) 2020 The Authors

This licence permits you to use, share, copy and redistribute the paper in any medium or any format provided that a full citation to the original paper in this journal is given, the use is not for commercial purposes and the paper is not changed in any way.
\end{abstract}


modalities. To date, conventional synchrotron-based computed tomography has been predominantly used in different healthy and pathologic models; meanwhile, spectral modalities such as deep ultraviolet (DUV) and infrared spectroscopies, and X-ray ptychography are beginning to provide new chemical, biochemical and 3D geometrical data about osteocyte lacuna, their surrounding matrix and its LCN.

\section{Focus on osteocyte biology and physiology}

Osteocytes, the main cell constituent of mammalian bones, represent more than $95 \%$ of all bone cells (20,000-80,000 cells $/ \mathrm{mm}^{3}$ in bone tissue). For a long time, the function of osteocytes was unknown and, consequently, their importance was underestimated. Over the last two decades, however, many publications have highlighted the role of this cell in bone homeostasis. Regarded for a long time simply as a cell at the end of its lifespan, the osteocyte is now considered as the initiator of the bone remodelling process. ${ }^{1}$ Indeed, osteocytes embedded in the mineral matrix form an interconnected network of cells in bone structure, which detect mechanical pressures and loadings.

Osteocytes have a dendritic morphology, while their cell body has a fusiform shape in long bones or sometimes rounded in flat bones. ${ }^{2}$ These cells are located in an osteocytic lacuna and have been buried in the matrix. ${ }^{3}$ They present some cytoplasmic extensions, dendrites, which extend into channels in the matrix called "canaliculi". Osteocytes communicate with each other and with the lining cells and osteoblasts at the surface of the bone tissue via these dendrites. ${ }^{4}$ The lacuna-canalicular system, which represents only $1 \%$ of interstitial fluid volume, constitutes a molecular exchange surface area which has been estimated at 400-fold higher than the Havers and Volkmann system and 133-fold higher than the trabecular bone system.

In the differentiation lineage, an osteocyte is an old osteoblast buried in the matrix that it has itself produced. Some of the pre-osteoblastic and osteoblastic biomarkers, such as osteopontin and integrin $\beta 3$, remain detectable in these cells. During the process of bone formation, some osteoblasts are left behind in the upwardly advancing newly formed osteoid material and become entombed in the matrix as an "osteoid osteocyte". During the process of burial, the future osteocyte maintains contact with the advancing osteoblasts and bone lining cells at the surface by extending dendrites, ${ }^{5}$ while the surrounding osteoid matrix becomes mineralised..$^{5}$ Aubin has suggested that only $10-20 \%$ of osteoblasts differentiate into osteocytes. $^{5}$

Cells at this early stage of osteoblast-to-osteocyte differentiation have been variously named as "large osteocytes", "young osteocytes", "osteoid osteocytes" or "preosteocytes". 6 They are larger than mature osteocytes and have numerous ribosomes, a well-developed endoplasmic reticulum and a wide Golgi complex, both involved in the synthesis of proteins and mucopolysaccharides. ${ }^{7}$ The study of the different differentiation markers allows for the profiling of osteocytes to determine the stage of cell maturation, i.e. young vs mature osteocyte. ${ }^{7}$ Osteocyte differentiation is accompanied by the progressive reduction of several bone markers (alkaline phosphatase, bone sialoprotein, osteocalcin, collagen type I, Runx2), the preservation of some markers (osteopontin, $\beta 3$ integrin, E11/gp38 antigen) and the appearance of new markers [CD44, dentin matrix protein 1 (DMP1), matrix extracellular phosphoglycoprotein (MEPE)] (see Figure 1). Once the osteoid mineralises, osteocyte ultrastructure undergoes further changes, including a reduction in the endoplasmic reticulum and Golgi apparatus corresponding to a decrease in protein synthesis and secretion. ${ }^{8}$ At this stage, many of the previously expressed bone markers are downregulated in the osteocyte (including alkaline phosphatase, bone sialoprotein, osteocalcin, collagen type I and Runx2). ${ }^{8}$

The osteocyte and its mechano-sensing properties are now considered as the cell at the centre of and the initiator of the bone remodelling process. ${ }^{1}$ Indeed, osteocytes form an interconnected network of cells having the capacity to detect mechanical pressures and loads.

Theoretical models and experiments strongly suggest that the lacuna-canalicular interstitial fluid flow varies with extra-vascular pressure and with variations of mechanical loading applied to bone tissue and osteocytes. ${ }^{9}$ Thus, mechanical forces applied to the bone provoke interstitial fluid movements along canaliculi and osteocyte lacunae, and, as a consequence, cause shear stress at the cell level and deformation of the osteocyte plasma membrane. ${ }^{10,11}$

Mechanical information might be directly detected by ciliar or flagellar structures of the cell membrane. ${ }^{12}$ 

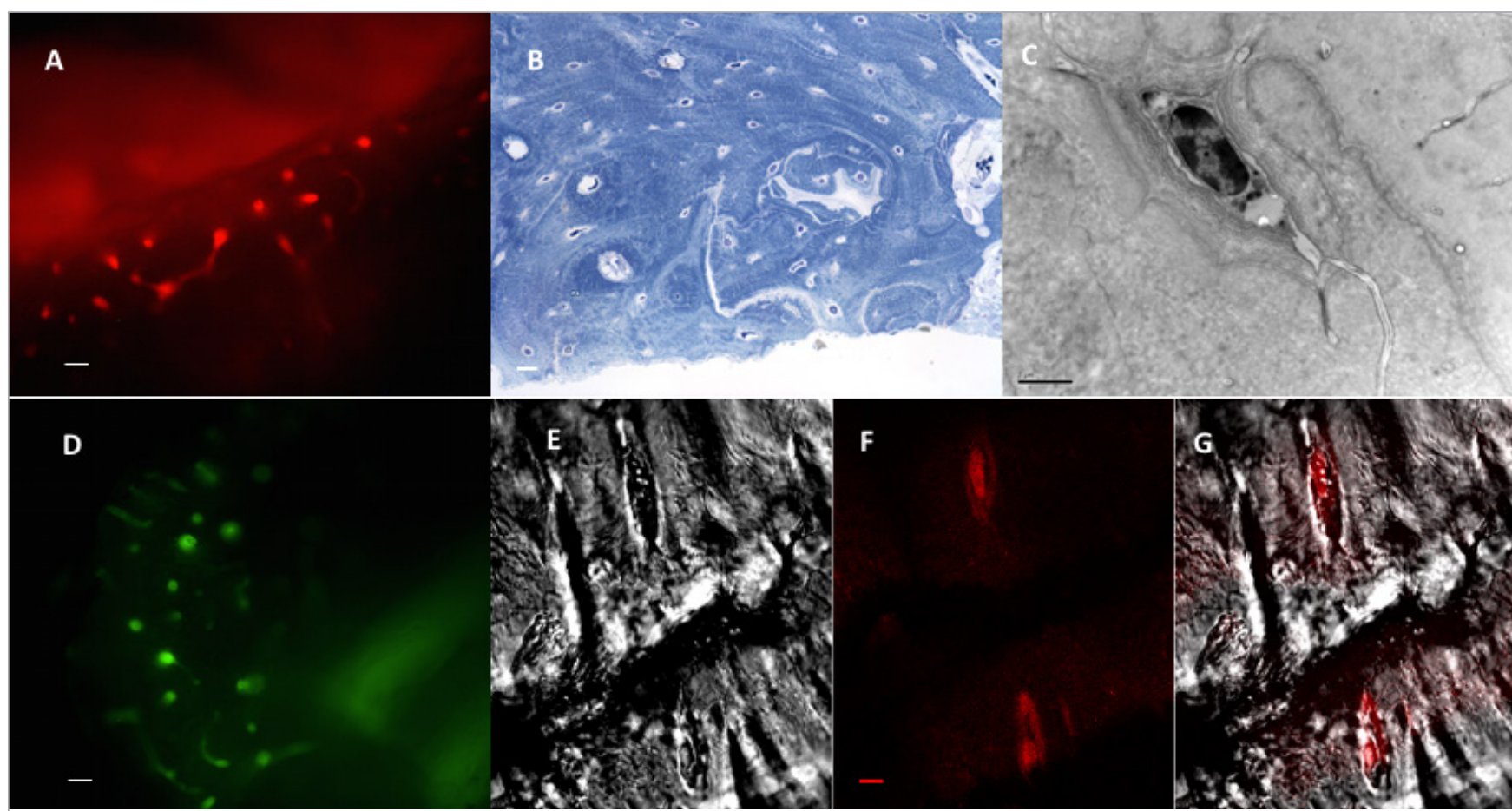

Figure 1. Conventional microscopic osteocyte imaging methods. A: epifluorescent microscopy-CD44 (differentiation marker) immunolabelling of the osteocyte in transversal cortical bone sections, in the upper half of the tibial diaphysis in an alcohol-induced osteoporosis rat model ( $\mathrm{PhD}$ of Delphine Maurel, Université d'Orléans, France) (white scale bar represents $10 \mu \mathrm{m})$. B: osteocytes lacunae observed with bright field microscopy on transversal tibial slices stained by toluidine blue in male Wistar rats. The objective magnification for the cortical bone was $\times 40$ (white scale bar represents $10 \mu \mathrm{m}$ ). C: osteocyte morphology in female Wistar rats by TEM (black scale bar represents $2 \mu \mathrm{m}$ ). The sections were then observed with a JEM 1011 electron microscope (Jeol, Tokyo, Japan) equipped with a Gatan digital camera driven by Digital Micrograph software (Gatan, Pleasanton, CA, USA). D: epifluorescent microscopy-phospho-paxilline immunolabelling of the osteocyte in transversal cortical bone sections, in the upper half of the tibial diaphysis in an alcohol-induced osteoporosis male Wistar rat model (PhD of Delphine Maurel, Université d'Orléans, France) (white scale bar represents $10 \mu \mathrm{m}$ ). E, F, G: confocal images of osteocytes were obtained on tibial slices (from male Wistar rat controls) fixed in formalin, cut with a high-speed rotatory tool (Dremel) and stained with a CD44 immunolabelling (middle image: F), a Zeiss Laser Scanning Microscope LSM 510 Meta confocal system attached to a Zeiss inverted microscope (Axiovert 200M; Zeiss) was used (40x oil immersion objective lens) (E: left image: bright field, G: right image: merge). The red scale bar represents $5 \mu \mathrm{m}$.

Whatever the mechanism of this mechano-reception, osteocytes are able to respond to mechanical stimulation by modulating the expression and the secretion of many molecules, including insulin-like growth factors (IGF-I and IGF-II), ${ }^{13}$ osteocalcin, ${ }^{13}$ sclerostin, ${ }^{14} \mathrm{c}$-fos, ${ }^{13}$ the synthesis prostaglandin enzymes $\mathrm{G} / \mathrm{H},{ }^{13}$ prostanoids $^{15}$ and nitric oxide (NO). ${ }^{16}$ Osteocyte mechano-reception may stimulate the Wnt/Lrp pathway as a negative regulator of sclerostin secretion, ${ }^{14}$ whereas the sclerostin itself is a negative regulator of the bone formation.

Osteocytes are cells that not only play a physiological role during their lifetime, but also achieve functions through their apoptosis. Micro-cracks have a deleterious effect on the bone tissue if they are in excess, with possibilities of micro-fissures, micro-fractures and fractures due to bone deficiency. However, the micro-cracks and the breaking of the lacunar network seem to play an important physiological role. ${ }^{17}$ It has been observed that pro-apoptotic molecules are elevated in osteocytes found in the vicinity of the micro-crack, whereas antiapoptotic molecules are expressed $1-2 \mathrm{~mm}$ from the micro-crack, ${ }^{17}$ suggesting that the apoptotic area may be restricted to the neighbourhood of the micro-crack.

The message transmitted by osteocyte apoptosis travels through the canalicular network to the surface of the bone tissue and is sent on to the progenitor cells. The real nature of this message is not known. It may consist of fluid movement, biochemical signals or electrical stimuli. This message leads to the initiation signals for remodelling. 
In phosphate and vitamin D metabolism, osteocytes produce $F G F 23$ in response to high rates of di-hydroxylated vitamin $\mathrm{D}$, and this production causes an inhibition of the renal reabsorption of phosphate from the distal tubule and a reduction in di-hydroxylated vitamin $\mathrm{D}$ production. ${ }^{18}$ In addition, the binding of osteocyte FGF23 on parathyroid glandular cells will induce a suppression of parathyroid hormone (PTH) secretion leading to increased calciuria and phosphaturia. ${ }^{19}$ In summary, FGF23 produced by osteocytes in response to increased rates of di-hydroxylated vitamin D will act on the kidneys and parathyroid gland through Klotho and lead to a decrease of phosphatemia.

An excess of FGF23 leads to a hypophosphatemia and can lead to an osteomalacia. FGF23 is secreted in excess by some mesenchymal tumours. ${ }^{20}$ Constitutively, FGF23 is mildly expressed by osteocytes and its synthesis is tightly controlled by the combined and inhibiting action of the DMP1 and PHEX proteins.

\section{Conventional ex vivo microscopic imaging methods}

Some of these methods are presented in Figure 1.

\section{Light microscopy}

"Standard" light microscopy (LM)

Much work has been carried out using LM on stained histological bone sections, which is fundamentally a 2D observation method. ${ }^{21,22}$ Indeed, an adequate spatial resolution of microstructural imaging techniques is needed to resolve the detail of the cell processes.

Nevertheless, specialised techniques such as reflected polarised LM have been used to quantify canaliculi. ${ }^{21}$ As in all light-based microscopy methods, the spatial resolution is restricted by the diffraction limit of visible light to around $200 \mathrm{~nm} .{ }^{21,23,24}$ The spatial resolution of LM is insufficient to image the osteocyte and the LCN completely, as the dendrites' diameters are around $100 \mathrm{~nm} .^{25}$

\section{Confocal laser scanning microscopy (CLSM)}

In CLSM, a point source laser light excites tissue which is either autofluorescent or has been stained with fluorescent dyes specific for cell components.

Stacking contiguous 2D optical sections produces 3D representations of internal bone microstructures. CLSM images have a spatial resolution of at best $200 \mathrm{~nm}$ in-plane and slightly worse (up to $450 \mathrm{~nm}$ ) out-of-plane. ${ }^{23,24}$ As in standard LM, this is not sufficient to image the osteocyte and LCN. The limitations of CLSM when imaging in dense mineralised tissue include the limited working distance (90-300 $\mu \mathrm{m})$ imposed by high-quality, high-numerical aperture objectives, and the decrease in signal with depth resulting from light absorption and scattering.

The practically achievable penetration depth of CLSM in mineralised tissue is $100-150 \mu m .^{26}$ The difference in resolution in the $x / y$-direction and the z-direction can also complicate the quantification and interpretation of 3D reconstructions. CLSM requires tissue to be cut, processed and cleared, thus departing from its native state. CLSM has been successfully used to characterise the spatial organisation, orientation and morphology of the osteocyte and LCN. ${ }^{2,27-29}$

Non-linear optical imaging methods devoted to intravital imaging of osteocytes

Due to their intrinsic optical sectioning and higher penetration depth compared to confocal microscopy, nonlinear optical imaging methods, such as two-photon excitation fluorescence (2PF) and second harmonic generation (SHG) microscopies, are increasingly used in bone research studies.

Moreover, 2PF microscopy has been previously used to show the LCN by in vivo calcein ${ }^{30}$ and rhodamine $B$ staining. ${ }^{31}$

Meanwhile, fluorophores have a tendency to photobleach, especially where high illumination power is necessary to clearly observe weak fluorescence signals such as those associated with canaliculi. Another strategy that recently evolved in microscopic imaging is the use of parametric processes, such as second and third harmonic generation, which do not require absorption and, moreover, do not provoke photochemical reactions leading to photobleaching.

Third harmonic generation microscopy is a very recent method devoted to non-invasive high-resolution osteocyte intravital imaging. ${ }^{31,32}$

It can be applied for high-resolution imaging of the LCN in calvaria in living mice. ${ }^{31}$ By performing third harmonic generation (THG) imaging in combination with two- and three-photon fluorescence microscopy, Torkaz et al. ${ }^{31}$ have shown that the THG signal produced from the bone-interstitial fluid boundary of the lacuna was higher 
than the THG signal from the interstitial fluid-osteocyte cell boundary.

Moreover, Torkaz et al. ${ }^{31}$ have used THG as a label-free imaging technique to examine structural variations in the LCN of live mice deficient in both histone deacetylase 4 and 5 (HDAC4, HDAC5). The three-dimensional analyses have revealed statistically significant differences between the HDAC4/5 double knockout and wild type mice in the number of osteocytes per volume and the number of canaliculi per lacunar surface area.

More recently, Genthial et al. ${ }^{32}$ have investigated the impact of microgravity on the 3D LCN structure in mice following space flight. They have built a specific analytical procedure to extract the LCN from THG images, which was described for ex vivo studies of femoral sections. ${ }^{32}$

We can consider that the use of THG microscopy thus offers a promising alternative to other methods suchas X-ray microtomography, which requires synchrotron radiation to achieve sufficient spatial resolution, and is shown to provide suitable data for quantitative and qualitative analysis in a biomedical context. Tokarz et al. have noted that the point spread function is near to $0.5 \mu \mathrm{m}$ laterally and $3.0 \mu \mathrm{m}$ axially; since canaliculi diameters $(95-550 \mathrm{~nm})$ are below the resolution of this microscope, canaliculi size cannot be determined with the Tokarz microscope. Data obtained by Genthial et al. ${ }^{32}$ suggested that the LCN was little modified by the microgravity during their used time scale (one month), they estimated that it was possible that this did not reflect changes in the osteocyte network. However, this inference may not be supportable given the spatial resolution limitation of the method.

\section{Electron microscopy}

Electron microscopy (EM) takes advantage of the shorter wavelength of electrons compared to visible light to extend resolving power. In practice, the best spatial resolution for biological tissue is near or below to $2 \mathrm{~nm}$ according to the EM method. ${ }^{33}$

\section{Transmission electron microscopy}

In transmission electron microscopy (TEM), electromagnetic lenses focus a beam of electrons which is transmitted through an ultrathin $(\leq 100 \mathrm{~nm})$ section of fixed, stained, dehydrated and resin-embedded tissue, where some electrons pass through the tissue and others are scattered to form an image. This has been the best technique for high-resolution imaging of osteocyte ultrastructure. For TEM, tissue must be prepared as ultrathin sections capable of withstanding low pressure and bombardment by high-energy electrons. This requires involved sample processing using chemicals and heat that can introduce artefacts such as shrinkage. Bone presents the further challenge of a hard, mineralised matrix requiring an additional step of decalcification to facilitate sectioning. Cryofixation is known to preserve tissue in a more life-like state, but has the drawback that only small ( $200 \mu \mathrm{m}$ deep) volumes of tissue can be preserved. It has been used to investigate tethering fibres between osteocytes and the pericellular matrix on a nanoscopic scale. ${ }^{25,34}$

Serial section TEM is technically difficult and time consuming, but provides high-resolution results, ${ }^{35,36}$ such as those used to elucidate the 3D shape of osteocytes. ${ }^{4}$ TEM tomography involves imaging a section repeatedly while it is incrementally tilted around its axis. ${ }^{37,38}$ The series of projections is used to reconstruct a 3D image of the sample. One disadvantage is the "missing wedge" caused by the limited tilt range of about $60^{\circ}$ in most TEMs. Ultra-high voltage electron microscopy (UHVEM) is not widely available and can only be used to image small samples (typically $3-5 \mu \mathrm{m}$ sections). ${ }^{39}$

Nevertheless, UHVEM studies have produced 3D images of osteocyte processes at $16 \mathrm{~nm}$ resolution ${ }^{40}$ and portions of the canaliculi down to around $2 \mathrm{~nm}$ resolution, providing significant information which contradicts previous studies. ${ }^{41}$

\section{Scanning electron microscopy}

The 2D technique of scanning electron microscopy (SEM) has been used to evaluate the number density of canaliculi in human bone. ${ }^{21}$ Resin-cast and acid-etched samples have been examined using SEM, showing the distribution, size and surface details of osteocyte lacunae.

\section{Volume SEM}

Serial section SEM is a complex technique where multiple ribbons of serial ultrathin sections of tissue were collected on a substrate and subsequently viewed by SEM. ${ }^{42}$ This technique is suitable for examining large areas at high $x$-y-resolution.

Both serial focused ion beam (FIB) SEM and serial block face (SBF) SEM produce high-resolution 3D data using automated sectioning techniques; $x / y$-resolution is comparable to TEM $(<5 \mathrm{~nm})$, whereas the $z$-resolution is limited by the sectioning technique, typically $10 \mathrm{~nm}$ for serial FIB SEM and $15 \mathrm{~nm}$ for SBF SEM. A 
proof-of-concept study by Schneider et al. for serial FIB SEM on bone tissue has successfully imaged parts of two adjacent osteocyte lacunae. ${ }^{33}$

\section{X-ray imaging}

Conventional X-ray computed tomography

Unlike light, $X$-rays penetrate bone easily and without causing image artefacts. Attenuation of $X$-rays by bone tissue provides image contrast for (2D) X-ray projections at different angular positions, which are used to reconstruct the 3D map of the specimen. Sample preparation requirements are minimal, the technique is non-destructive and once set up, operator involvement is low. Bone can be imaged and analysed at different levels (organ, tissue, cell) using micro-computed tomography $(\mu \mathrm{CT})$ at isotropic resolutions down to $1 \mu \mathrm{m}$ and below. ${ }^{43}$

Lab-based X-ray $\mu C T$ systems do not reach the resolution of EM. Thus, $\mu C T$ allows visualisation of lacunae, but not the fine detail of canaliculi. Commercially available $\mu \mathrm{CT}$ systems with improved spatial resolution have recently become available, some of which claim voxel sizes in the order of $50 \mathrm{~nm}$ (e.g., ZEISS Xradia 810 Ultra). Studies using this equipment have investigated differences in morphology of lacunae from different sites in the mouse ${ }^{2}$ and compared morphology of lacunae in humans with different bone diseases, where significant variations have been observed between osteoarthritis, osteopenia and osteopetrosis. ${ }^{2,44}$ A drawback of CT at these particular low voxel sizes below the micrometre range is the long acquisition time of many hours.

\section{Synchrotron radiation imaging methods that have been used to study osteocytes}

\section{$\mathrm{X}$-ray synchrotron radiation computed tomography}

Synchrotron radiation (SR) sources, operated as largescale electron accelerators at a small number of sites worldwide, offer quasi-monochromatic $X$-rays and much higher photon fluxes with small $X$-ray source spot sizes, providing higher spatial resolution than conventional micro-CT which are sufficient to resolve trabecular and internal bone microstructures at the micrometric scale. ${ }^{45}$

Contrast absorption SR-CT has been used to investigate trabecular and cortical bone micro-structure ${ }^{46,47}$ and osteocyte lacunae. ${ }^{48,49}$ Thanks to the monochromaticity of an X-ray beam, there is no beam hardening artefact, the result is that images can be considered as a map of the linear attenuation coefficient within the sample. Consequently, the attenuation coefficient can be easily used to estimate the degree of mineralisation of bone. In addition to X-ray absorption-based $\mathrm{CT}$, coherent synchrotron light allows to can also image the internal properties of bone with a higher spatial resolution and intensity than laboratory-based techniques because the system can fully exploit phase contrast. ${ }^{50,51}$ Phase contrast takes advantage of the fact that bone, marrow and air have different refractive indices.

The technique is particularly useful for enhancing the contrast of surfaces and interfaces in samples, which would not be visible using conventional absorption CT.

Phase-contrast SR-CT is the only suitable nondestructive 3D imaging technique for visualising and quantifying micro-damage of bone in high resolution without any contrast agents. To obtain phase contrast at very high resolution, three systems can be used, the easiest to implement is in-line propagated based $X$-ray imaging, the second one is ptychography and the third is Talbot interferometry. In holotomography the acquisition of the phase $\mathrm{CT}$ images consists of recording several scans at different detector distances. However, in some cases, single distance phase can be used by employing the frequency filtering method..$^{52}$ Indeed, with this latter method and a voxel size of $760 \mathrm{~nm}$, it was possible to assess the morphology, the 3D distribution and propagation of micro-cracks under different loading conditions in a large sample size $(>3 \mathrm{~mm}),{ }^{53}$ while confocal microscopy can be used to produce 3D images of micro-damage but only at a depth of about $200 \mu \mathrm{m}$. In addition, the observation of the $3 \mathrm{D}$ organisation of the LCN was obtained with a voxel size about $60 \mathrm{~nm}$ based on acquisitions at four different focal spots. ${ }^{54}$

\section{Synchrotron ptychographic X-ray CT}

Ptychographic X-ray nanotomography (PXCT) exploits the penetrating power of hard $\mathrm{X}$-ray diffraction patterns, it is a coherent diffraction microscopy, or "lens-less imaging" system, that produces absorption and phase-contrast images, which can be directly converted into quantitative $3 \mathrm{D}$ images of the electron density within objects. ${ }^{55}$ With this new tool, it was possible to characterise the LCN (Figure 2) with a resolution of tens of nanometres, for bone specimens up to $100 \mu \mathrm{m}$ in diameter. 


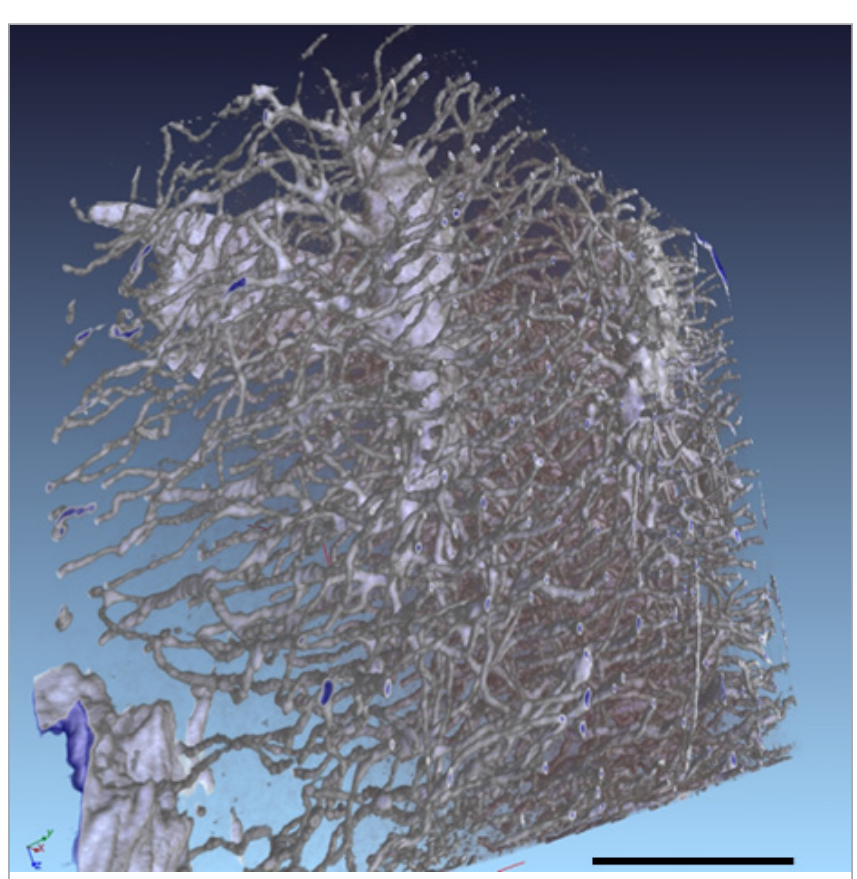

Figure 2. Ptychographic osteocyte and LCN imaging. Three-dimensional iso-surface rendering of the internal morphology of a sample of cortical rat bone from the tibial diaphysis, showing the lacuna-canalicular network around at least two osteocytes. This structure is revealed non-destructively, by segmenting a quantitative 3D electron density map with voxel size of $44 \mathrm{~nm}^{3}$ that has been reconstructed from ptychographic X-ray coherent diffraction tomography data ${ }^{55}$ obtained at the SLS, cSAXS beamline. The black scale bar represents $10 \mu \mathrm{m}$.

The last setup to provide phase contrast is to use a Talbot interferometer consisting of two gratings, one phase grating, placed after the sample, and an analyser, placed at the "Talbot distance" behind the phase grating, respectively. Acquisition is performed with the interferometer, and again without after shifting the lens downstream. ${ }^{56}$ Grating-based phase-contrast has been used to analyse the degree of bone mineralisation in mouse cortical bone around the LCN.

\section{Synchrotron DUV fluorescence spectroscopic microscopy}

While most biomolecules do present a contrast in DUV transmission microscopy, a few of them will fluoresce. Autofluorescence is important to contribute to a better discrimination of molecules. Furthermore, directly following autofluorescence opens the possibility to conduct label-free studies of molecules of interest without any external probes which could impair activity of the molecule of interest. 57,58
While many studies were conducted with DUV fluorescence spectroscopy as a diagnosis tool, mainly for cancer, ${ }^{59}$ very few were conducted on DUV fluorescence microscopy or micro-spectrofluorimetry on biopsies and tissues. Most of them used excitation wavelengths longer than $350 \mathrm{~nm}$, mostly due to the lack of laser lines below this value, despite very promising sensibility and sensitivity using DUV excitation. ${ }^{60}$ Indeed, many endogenous fluorophores have an absorption/excitability maximum in the DUV below $350 \mathrm{~nm}$, especially tryptophan. ${ }^{59}$

To assess bone quality (bone micro-architecture, collagen content) at subcellular resolution, synchrotron DUV spectroscopy can give information about the biochemical content of the osteocyte lacunae and their surrounding matrix, osteocytes being considered as the orchestrator of bone remodelling. ${ }^{61}$ In a rat experimental model of alcohol-induced osteoporosis, osteocytes lacunae/surrounding matrix autofluorescence images were investigated of the cortical bone of the tibia. ${ }^{62}$

Synchrotron UV micro-spectroscopy was performed at the DISCO beamline at the SOLEIL SR facility (SaintAubin, France). Monochromatised UV light (typically between $270 \mathrm{~nm}$ and $330 \mathrm{~nm}$ ) was used to excite cortical tibial sections through a 40x ultrafluar objective (Ultrafluar, Zeiss, Germany). The fluorescence emission spectrum arising from each excited pixel is recorded. Raster-scanning of the sample allows one to record $x, y$, I, I maps of interest. Mapping of $20 \times 20 \mu \mathrm{m}^{2}$ was performed with a $2 \times 2 \mu \mathrm{m}$ step size: the step size was chosen to provide a field of view containing several cells with a $5 \mathrm{~s}$ acquisition time per spectrum. Regions of Interest (ROIs) were selected, both centred around an osteocyte and in the surrounding matrix. For each rat sample, three maps were measured, containing both osteocyte ROIs and matrix ROIs. Each map was, therefore, a matrix of 100 spectra from which noise and spikes were removed using a home-made routine in MATLAB (Mathworks Inc.) available at DISCO beamline. The spectra were classified into subsets corresponding to osteocyte or matrix, using masks created from the transmission images (Figure 3).

Thereafter, all spectra from all ROls were loaded in Igor Pro (Wavemetrics Inc.), the baselines were zeroed and the integral of the three peaks were measured as follows: area 1 for tyrosine (290-317 nm), area 2 for tryptophan (322-370 nm) and area 3 for collagen (380-440 nm). The collagen signal originates from the cross-links between the amino acids. 


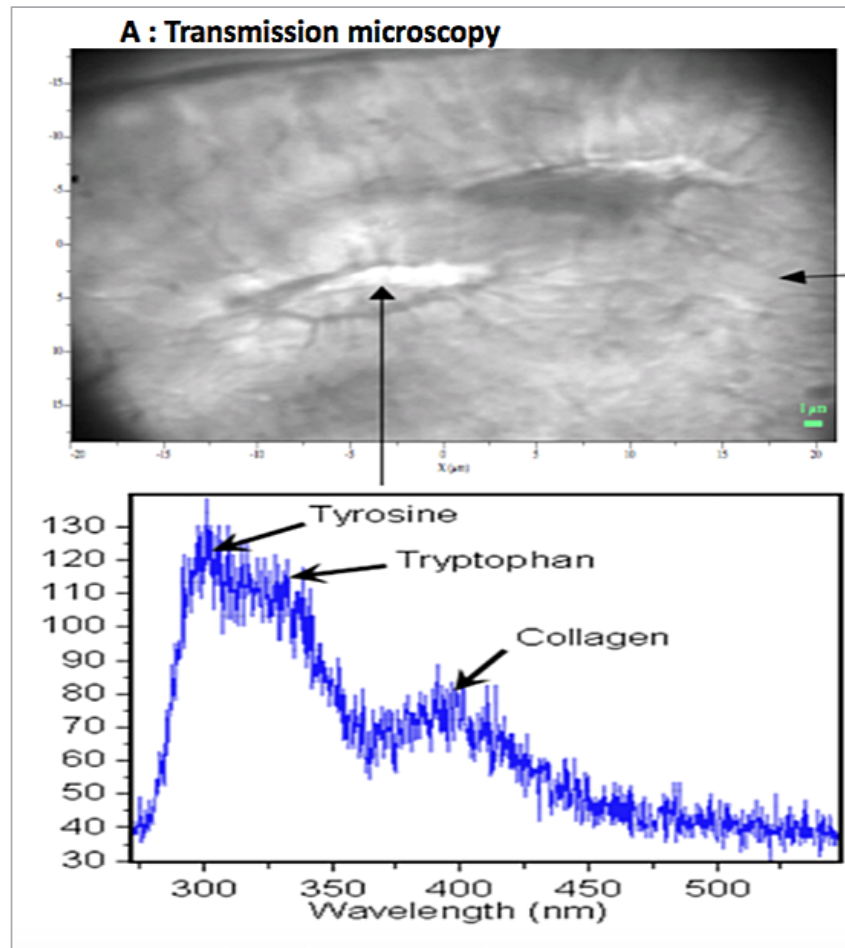

C : UV intensity spectrum in osteocyte
B : UV intensity spectrum in matrix

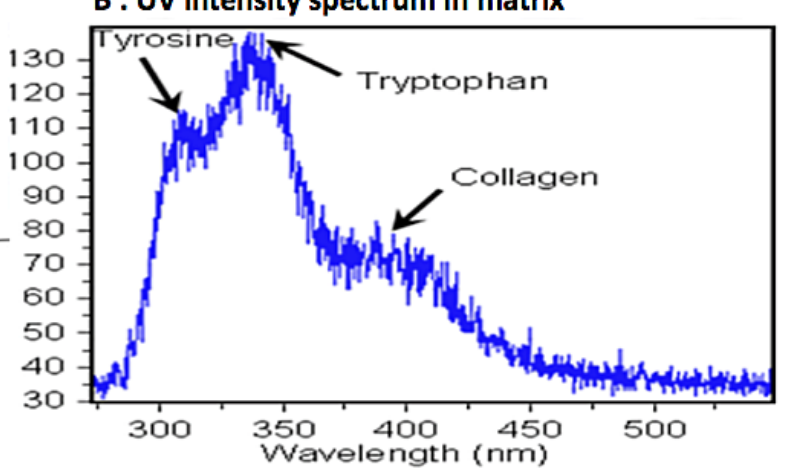

\begin{tabular}{|l|l|l|l|}
\hline & $\begin{array}{l}\text { Tyr/Coll matrix } \\
\text { ratio }\end{array}$ & $\begin{array}{l}\text { Tyr/Coll } \\
\text { osteocyte ratio }\end{array}$ & $p$ \\
\hline $\begin{array}{l}\text { Control } \\
\text { samples }(n=11)\end{array}$ & $2.28+/-1.23$ & $2.30+/-1.44$ & NS \\
\hline & $\begin{array}{l}\text { Trp/Coll matrix } \\
\text { ratio }\end{array}$ & $\begin{array}{l}\text { Trp/Coll } \\
\text { osteocyte ratio }\end{array}$ & $p$ \\
\hline $\begin{array}{l}\text { Control } \\
\text { samples }(n=11)\end{array}$ & $4.44+/-2.32$ & $3.93+/-2.27$ & 0.02 \\
\hline
\end{tabular}

D : UV spectroscopy Tyrosine/ Collagen (Tyr/Coll) and Tryptophan/Collagen (Trp/Coll) ratios in osteocyte and matrix in control samples

Figure 3. UV spectroscopy results from osteocyte and surrounding matrix ROI. A: transmission microscopy image acquired at the DISCO beamline, Synchrotron SOLEIL, showing ROIs. B: UV intensity spectrum originating from one matrix ROI pixel (indicated with an arrow). C: UV intensity spectrum originating from one osteocyte ROI pixel as shown. D: UV spectroscopy Tyrosine/Collagen (Tyr/Coll) and Tryptophan/Collagen (Trp/Coll) mean ratios \pm SD in osteocyte and matrix acquired in the cortical part of tibial diaphysis, in male Wistar rat (age: 30 weeks) control samples $(n=11)$, NS: not significant, the critical $p$-value for statistical significance was $p=0.05$.

For each mapping, the ratios tyrosine/collagen (Tyr/ Coll), tryptophan/collagen (Trp/Coll) and tyrosine/ tryptophan (Tyr/Trp) were calculated. The duration of each mapping was $8 \mathrm{~min}$. The sensitivity of UV microspectroscopy on tissue was on the order of micromolar. Autofluorescence spectra were deconvoluted using Labspec software (Jobin-Yvon, France).

The fluorescence emission spectra present three peaks situated at $305 \mathrm{~nm}, 333 \mathrm{~nm}$ and $385 \mathrm{~nm}$, attributed to tyrosine, tryptophan and collagen, respectively. The ratio between tyrosine and tryptophan is dependent on the site in the cortical bone tissue (osteocyte lacuna vs surrounding matrix) (Figure 3). From our knowledge, no other tissue presents a similar high level of tyrosine fluorescence.

\section{Synchrotron FT-IR spectroscopic imaging}

Fourier transform infrared micro-spectroscopy (FTIRM) is commonly used to investigate the structural features of organic molecules found in bone by examining the frequency at which molecular bonds vibrate (i.e. stretching and bending). ${ }^{63-65}$ Moreover, vibrational spectroscopy in the mid-infrared (IR) region provides molecular structure information about mineralised and non-mineralised connective tissue. ${ }^{66}$ In addition, the FTIRM system, in which the spectrometer is coupled to a light microscope, enables investigators to record spectra at discrete points within a thin tissue section. This methodology has several advantages compared to other approaches such as X-ray diffraction, nuclear magnetic resonance or high-performance liquid chromatography. First, it allows the use of the same sample for other investigations (histomorphometry, quantitative backscattered electron imaging, nanoindentation) and, second, it enables a precise investigation of tissue material properties in a specific location of the bone sample (between double tetracycline labels as for example).

In previous work, ${ }^{67}$ tibial sections were embedded in polymethylmethacrylate resin to prepare samples to be adapted to synchrotron infrared excitation on the SMIS 
beamline at the Synchrotron SOLEIL. The photon energy range requested is $2200-3000 \mathrm{~cm}^{-1}$.

For IR SR requested on the SMIS beamline, the authors needed a subcellular high resolution to achieve a mapping step of $6 \times 6 \mu m^{2}$ to obtain specific chemical information in osteocyte lacunae. ${ }^{67}$ Resolution is limited in a conventional source by the brightness of the source between $15 \mu \mathrm{m}$ and $150 \mu \mathrm{m}$, thanks to the synchrotron source, by the diffraction, the resolution is improved to between $3 \mu \mathrm{m}$ and $12 \mu \mathrm{m}$, in the mid-infrared.

On this beamline, the acquisition mode was transreflection. Bone pieces were placed on IR-transparent windows such as $\mathrm{BaF}_{2}$ or $\mathrm{ZnS}$. The spectral resolution used was $4 \mathrm{~cm}^{-1}$. The spatial resolution was adapted to discriminate osteocyte lacunae and bone matrix: $6 \times 6 \mu^{2}$ to obtain points in the osteocyte lacunae, and obviously a greater number of points acquired in their surrounding matrix. A minimum of six acquisitions for each rat in each experimental group (alcohol-induced osteoporotic group versus control), for both the osteocyte lacunae and the matrix needed to be imaged to obtain statistically valid results. The post-treatment analysis, chemical mapping and area ratios cwere obtained with Omnic-Atlus software (Thermo Fisher Scientific).

\section{Results and discussion Synchrotron-based CT results applied to human bone samples}

$\mathrm{SR}$ micro-CT is a technology which provides highly accurate $3 \mathrm{D}$ images for analysis at micrometre or better resolutions. SR micro-CT has been utilised to examine human bone and has the ability to explore a large field of view. ${ }^{54}$ The main advantages compared to standard $\mathrm{CT}$ are that it can offer a high signal-to-noise ratio, higher spatial resolution $^{45}$ and monochromaticity facilitating segmentation and quantitative analysis. Moreover, the high photon flux allows a significant decrease of the scanning time ${ }^{68}$ and no advanced staining and sample preparations are necessary. The main parameters used are presented in Table 1.

Results obtained in healthy bones samples

Carter et al. ${ }^{69}$ have achieved SR micro-CT acquisitions (Argonne 2BM) with a voxel size of $1.47 \mu \mathrm{m}$ at an energy of $27.9 \mathrm{keV}$.

The most significant results were the reduction in lacunar volume in the older women, when dividing the subjects into groups of women younger or older than 50 years. The osteocyte lacunar volumes were approximately $30 \%$ larger in the younger than in the older women with no significant changes in osteocyte lacunar density according to age. ${ }^{69}$ Under 50 years old, bone is characterised in flatter and less circular lacunae than those of the older group which are more equal in dimensions. $^{70}$

With the same acquisition parameters, they compared four quadrants (anterior, posterior, medial and lateral) in seven mid-cortical femoral diaphysis. They found a dramatic difference of $54 \%$ in lacunar density between the anterior and medial region. ${ }^{70} \mathrm{~A}$ preliminary study from the same group at $26.4 \mathrm{keV}$, studying one individual found differences between medial and anterior/posterior regions. ${ }^{71}$ Lacunae from the anterior region were more elongated and less flat than those from the medial and lateral regions. Lacunae from the posterior region were significantly more elongated and less flat than those from the medial, but not the lateral regions. ${ }^{70}$ This study demonstrated a lower number of more elongated spaces along the anterolateral axis and an increase in less elongated but flatter lacunae in the tension and compression zones (lateral-medial). ${ }^{70}$ Anterior blocks from the middiaphysis of 30 women aged from 20 to 86 years were studied. No significant variation of lacunar density was found according to age, but a significant reduction in volume of lacunae was observed. ${ }^{71}$

Hannah et al. ${ }^{72}$ achieved SR micro-CT acquisitions of the femoral diaphysis (voxel size of $1.47 \mu \mathrm{m}$ ), at the energy of $26.4 \mathrm{keV}$ (Argonne 2BM). Osteocyte density varied from 40,000 per $\mathrm{mm}^{3}$ close to the Haversian canals to about 900,000 per $\mathrm{m}^{3}$ at $80 \%$ of the osteon radius. ${ }^{72}$ They found two distinct peaks of osteocyte lacunae, one at $200 \mu^{3}$ and one at $330 \mu m^{3}$. The distance between lacunae was an average of $23 \mu \mathrm{m} .^{72}$

Bash-Gansmo et al. ${ }^{73}$ studied the osteocyte lacunar properties of the iliac crest cortex according to age and sex, at the Swiss Light Source (TOMCAT beamline), at a voxel size of $0.65 \mu \mathrm{m}$, and at the energy of $35 \mathrm{keV}$. They found that the lacunar volume was unaffected by both age and sex, the osteocyte density did not differ between men and women with only a significant decrease with age when pooling data from both sexes. ${ }^{73}$

Dong et al. ${ }^{74}$ carried out SR micro-CT at the European Synchrotron Radiation Facility (ESRF, Grenoble, France) ID19 beamline, with a voxel size of $1.40 \mu \mathrm{m}$. In 13 cortical mid-diaphysis cortical samples from female 


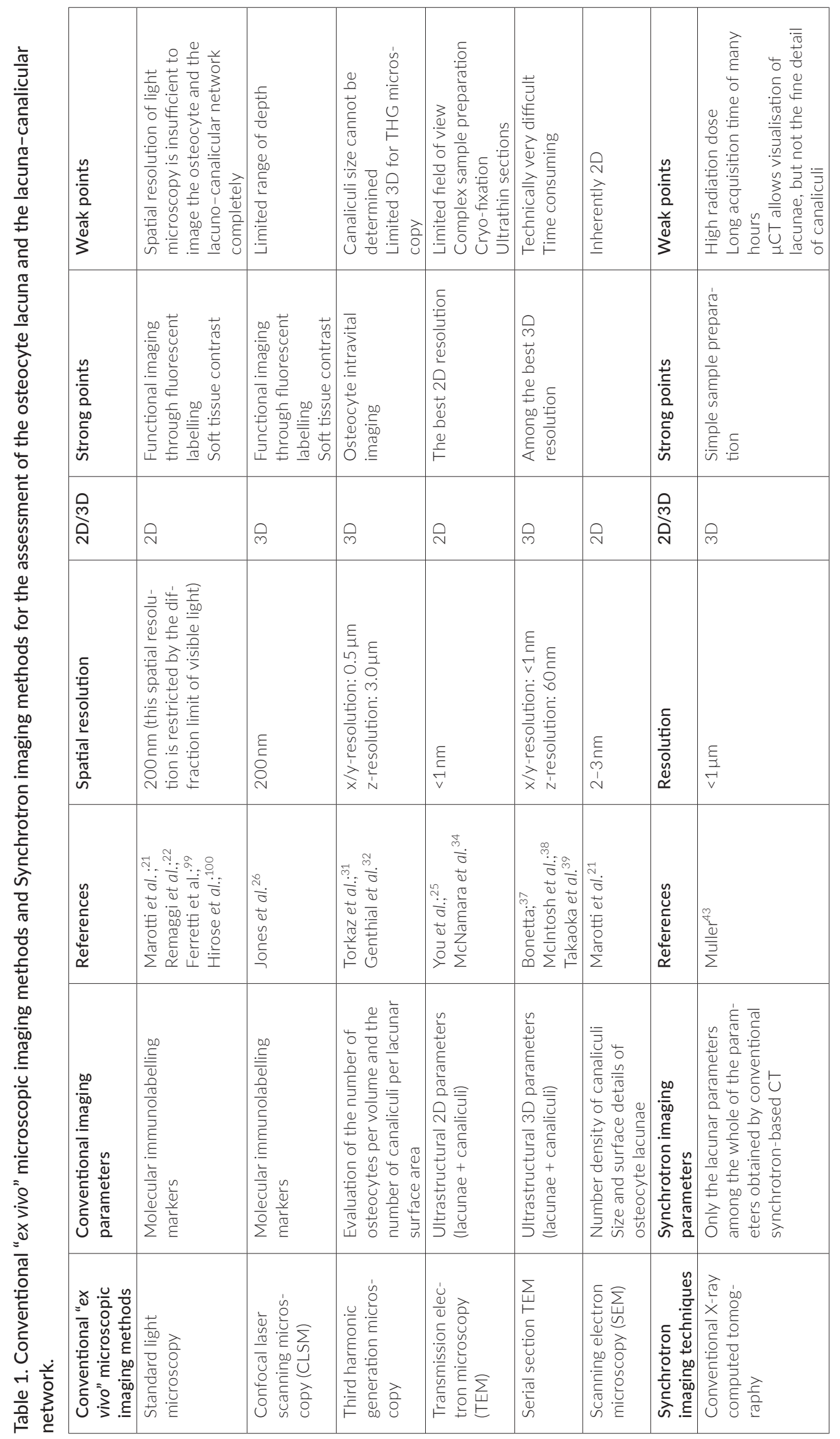




\begin{tabular}{|c|c|c|}
\hline 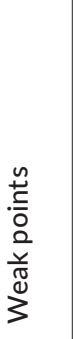 & 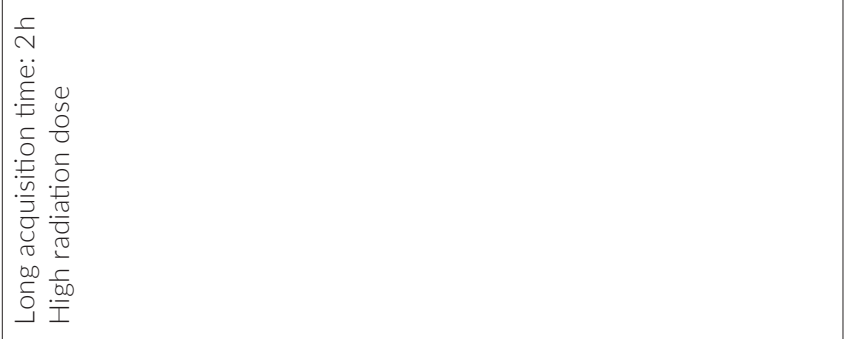 & 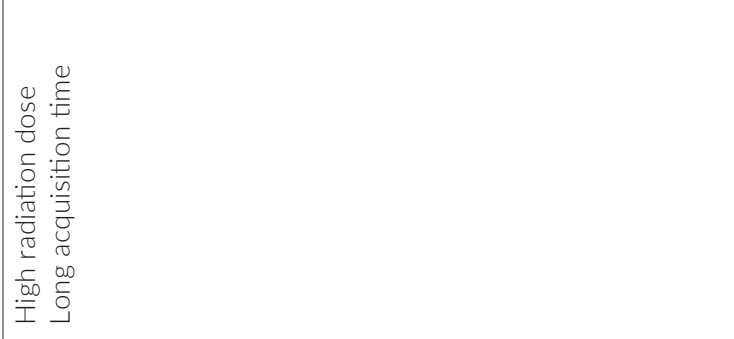 \\
\hline 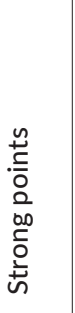 & 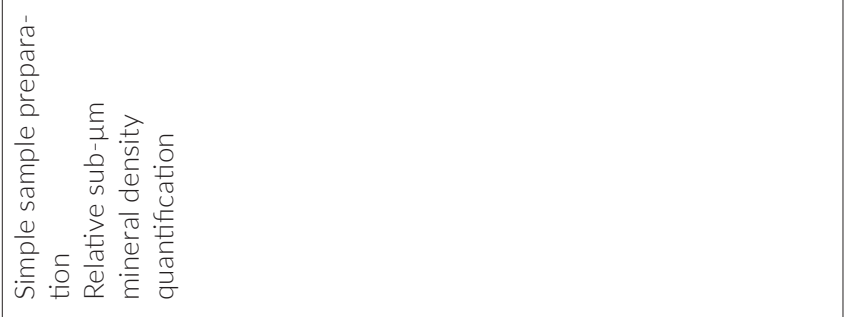 & 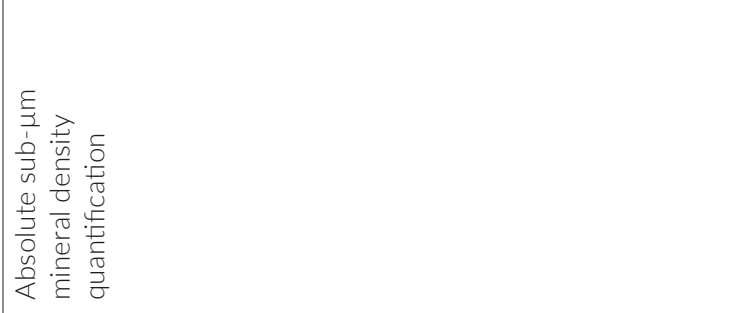 \\
\hline$\stackrel{\text { Ò }}{\text { à }}$ & ळे & pे \\
\hline & 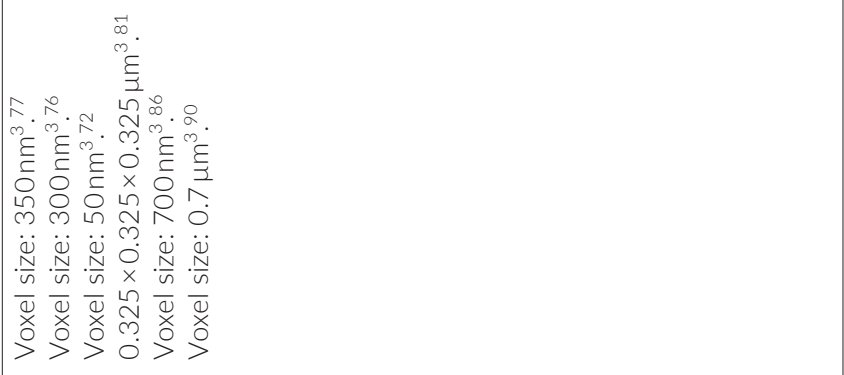 & 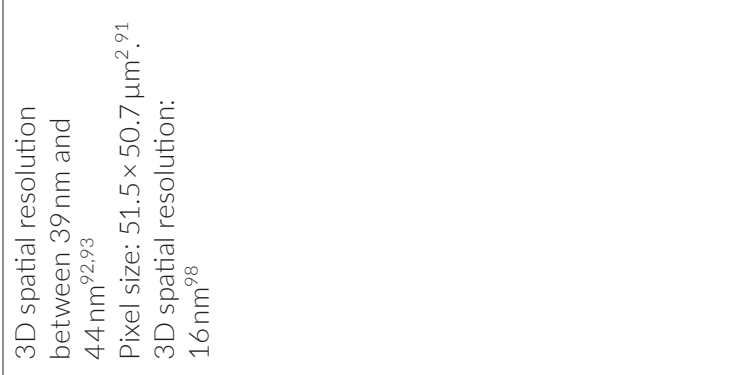 \\
\hline 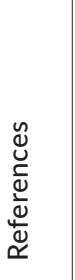 & 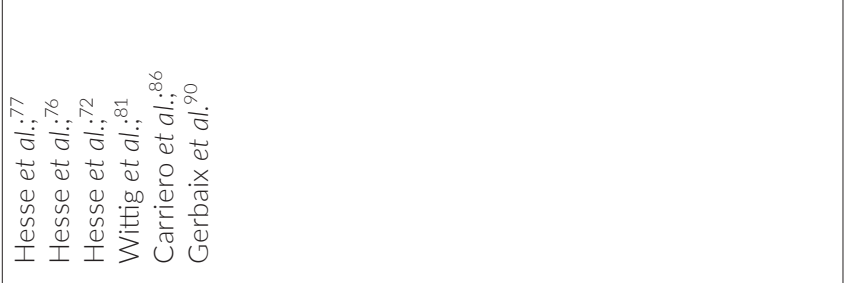 & 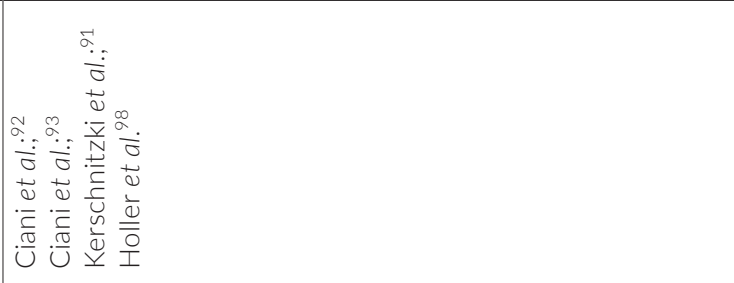 \\
\hline 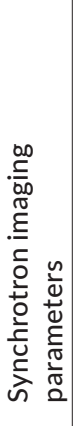 & 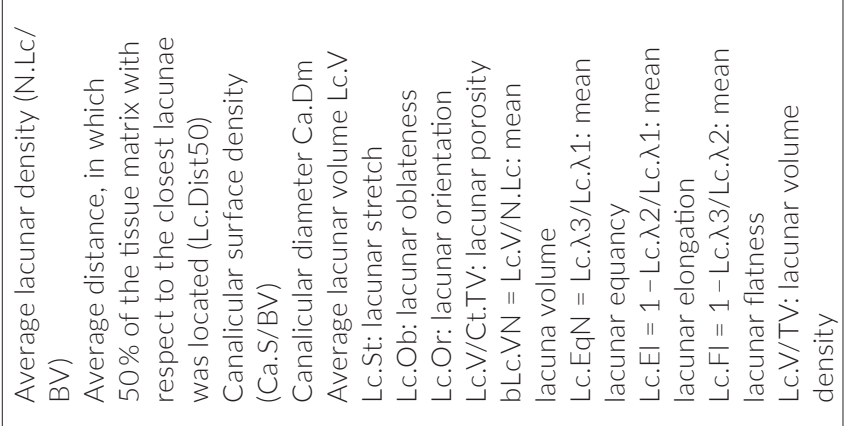 & 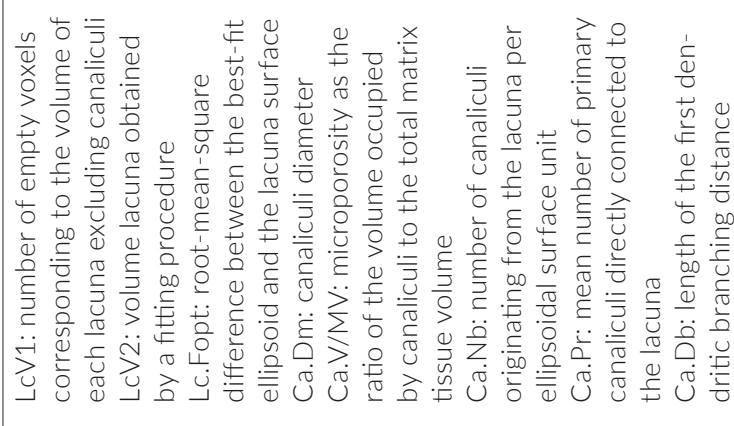 \\
\hline 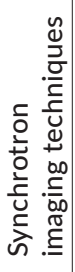 & 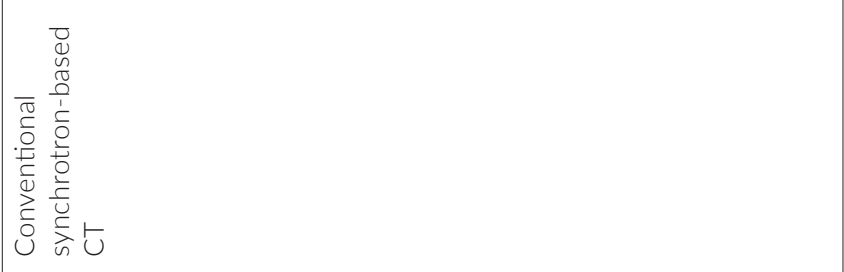 & 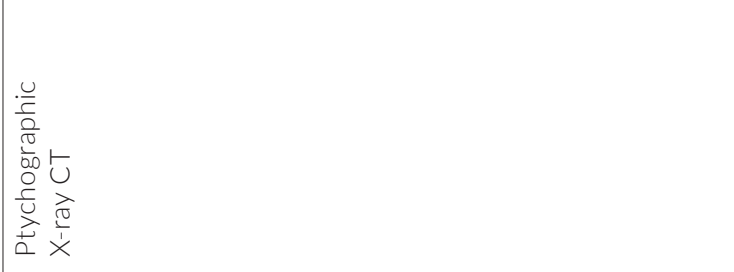 \\
\hline
\end{tabular}




\begin{tabular}{|c|c|c|}
\hline & 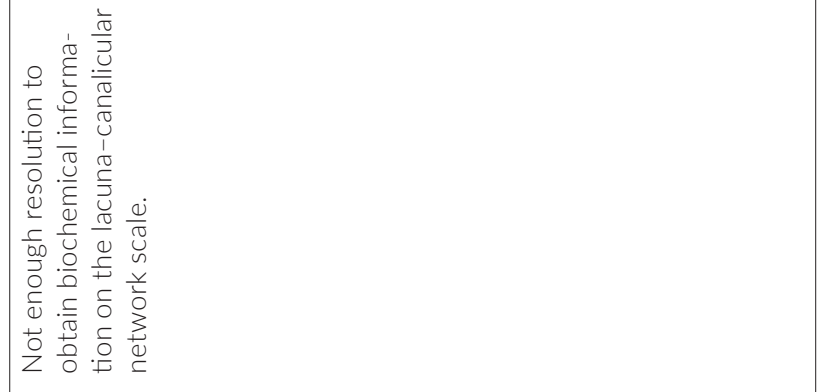 & 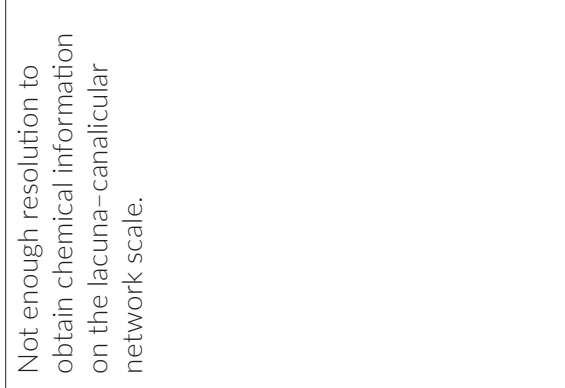 \\
\hline 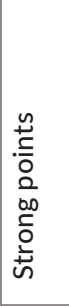 & 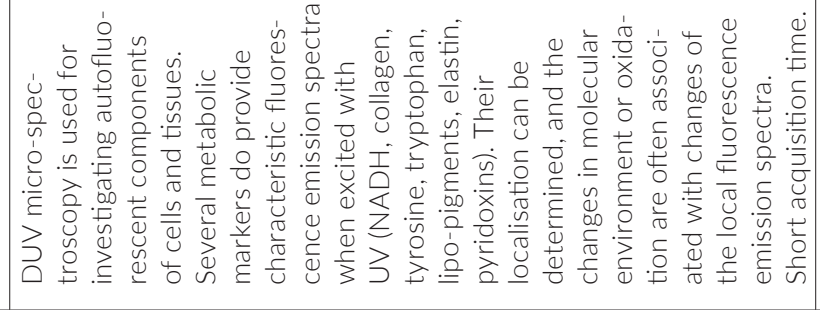 & 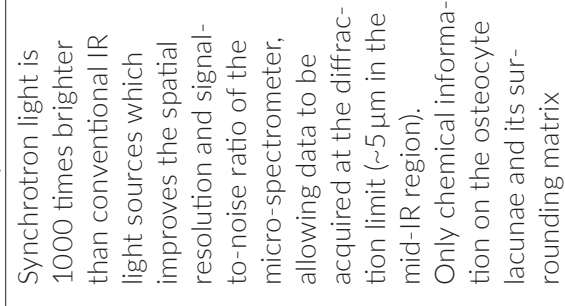 \\
\hline $\begin{array}{l}\stackrel{\rho}{\rho} \\
\text { ì }\end{array}$ & $\stackrel{\sim}{\sim}$ & 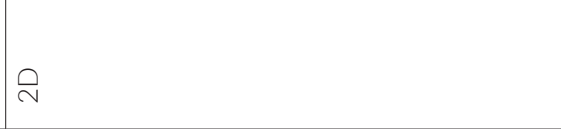 \\
\hline 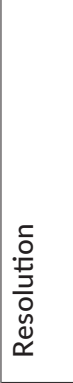 & 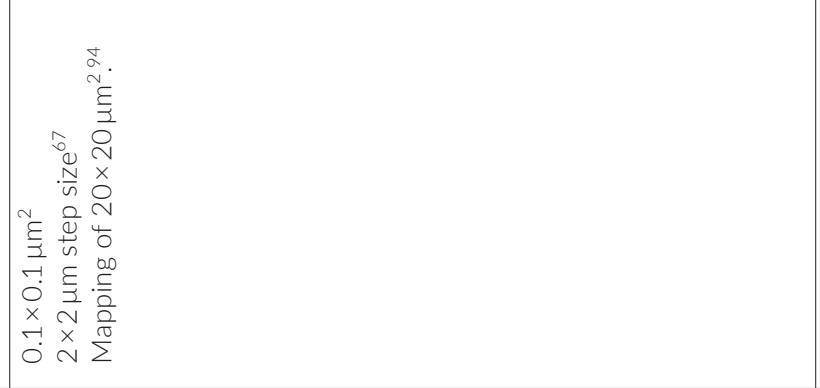 & 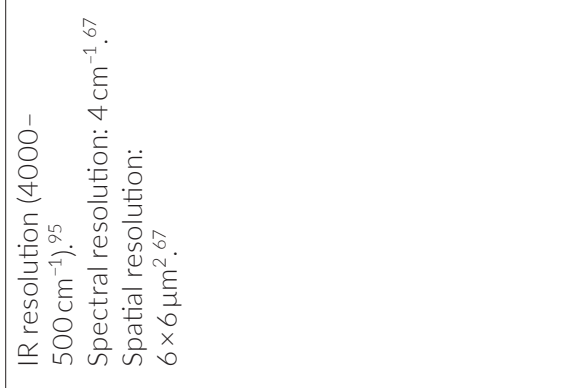 \\
\hline 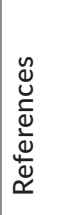 & 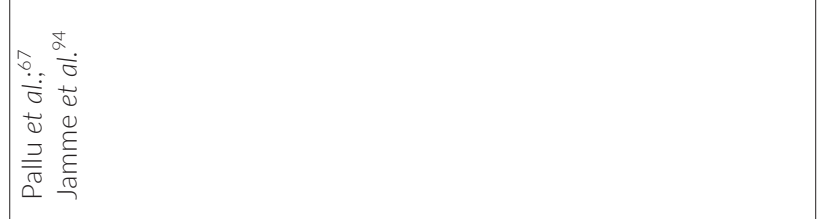 & 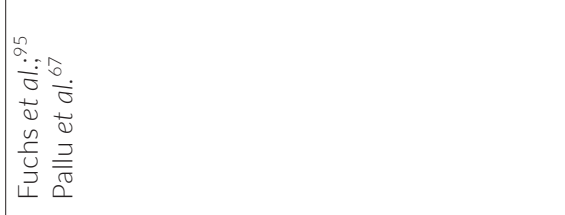 \\
\hline 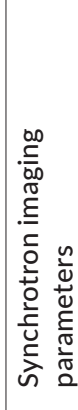 & 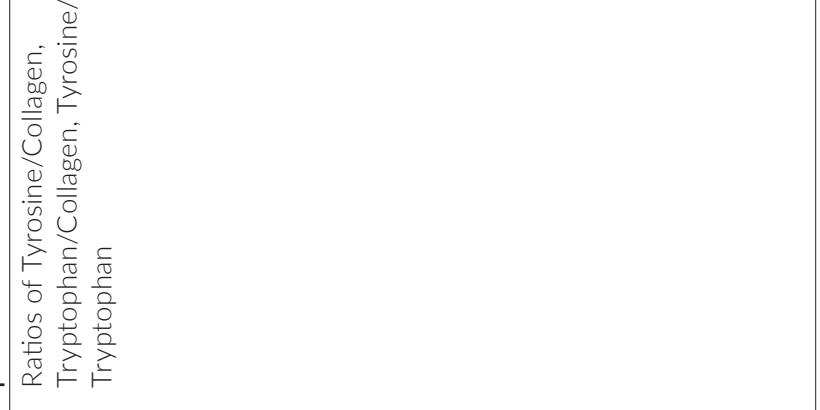 & 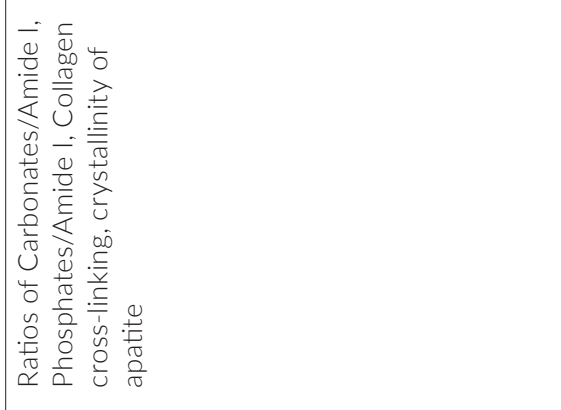 \\
\hline 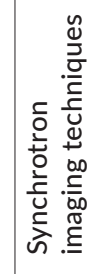 & 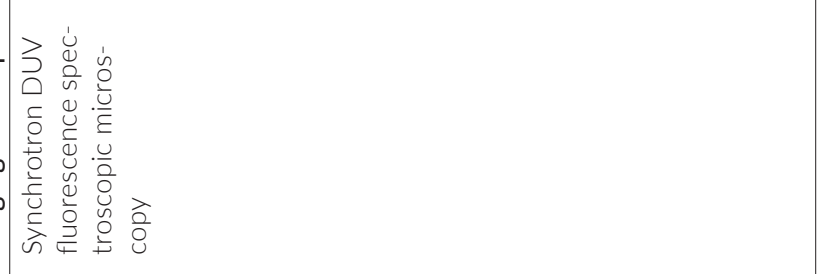 & 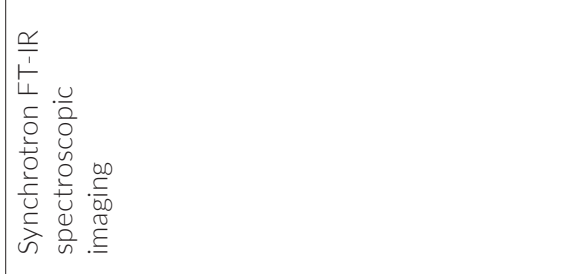 \\
\hline
\end{tabular}


donors without precision of the measurement sites, the mean volume was found to be $409 \pm 150 \mu \mathrm{m}^{3}$ with average dimension length $18.9 \pm 4.9,9.2 \pm 2.1 \mu \mathrm{m}$ in width, $4.8 \pm 1.1 \mu \mathrm{m}$ in depth. ${ }^{74}$ The main direction of each osteocyte was in the direction of the Haversian canals. ${ }^{74}$ Lacunar number density, the three axes of the lacunae and the anisotropy of lacunae were significantly correlated with Bone Volume/Tissue Volume (BV/TV) \% and, in the most porous region, the increase of anisotropy was mainly due to the increase in length of lacunae while the width and depth slightly decreased. ${ }^{74}$

To study the weight bearing influence on the 3D lacunar morphology, Gautier et al. studied paired femoral diaphysis, neck and radius at $0.7 \mu \mathrm{m}$ of resolution from eight female donors. ${ }^{68}$ They achieved SR micro-CT acquisitions at ESRF ID19 at a voxel size of $0.70 \mu \mathrm{m}$, at an energy of $31 \mathrm{keV}$.

The lacunar density was 18\% lower in the radial diaphysis than in the femoral neck, the amount of lacunae within the femoral neck was significantly higher than in femoral or radial diaphysis. Shapes of lacunae in the radius were sharp (with Lc.L1 and Lc.L2 lower) than the femoral diaphysis and femoral neck whereas no difference for Lc.V between the three locations. ${ }^{68}$

Langer et al. ${ }^{54}$ studied the LCN structure in middiaphysis of two female donors with phase contrast. They achieved SR micro-CT acquisitions at ESRF ID22NI at a voxel size of $60 \mathrm{~nm}$, at an energy of $17 \mathrm{keV}$. The main finding was that the lacunae had similar size and shape in osteonal and were highly connected in osteons, whereas in the interstitial tissue all the lacunae were disconnected with variable size and shape. ${ }^{54}$

\section{Results obtained in bone physio-pathological context}

\section{Osteonecrosis}

In the most recent study by Hesse et al., ${ }^{75}$ bone samples were extracted from human female jawbone sections originating from four healthy subjects (aged 19, 40, 42 and 47 years) and four patients suffering from Bisphosphonate-Related Osteonecrosis of the Jaw (BRONJ) (aged 40, 70 and 72 years). The aim of the study was to investigate the spatial distribution of mass density in the perilacunar and pericanalicular bone matrix and to explore how these densities are influenced by tissue aging. Phase nano-computed tomography (PNCT) measurements were carried out on beamline ID 22NI at the ESRF at four distances between focus and sample.
The voxel size was $50 \mathrm{~nm}$. The energy of the beam was set to $16.874 \mathrm{keV}$.

Average canalicular porosity was found to be around $2 \%$, with large variability between the individual volumes of interest (VOIs). The average Ca.Dm, Ca.S/BV and Ca.Dist50 (distance to canaliculi boundary in which $50 \%$ of $\mathrm{BV}$ is located) of all analysed VOls were found to

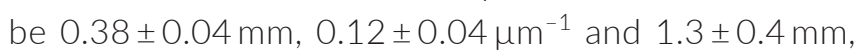
respectively. Furthermore, the canalicular surface density (Ca.S/BV) showed significant correlation with Ca.V/BV, Ca.Dm and Ca.Dist50. ${ }^{75}$ Their results provide evidence that mass density results in negative gradients with respect to the distance from both lacunae and canaliculi, which diminish with increasing tissue age in healthy bone. The values for the lacunar volumes tend to be higher than those reported previously for similar samples at a lower imaging resolution, suggesting that the spatial resolution and segmentation methods of the imaging setup have a strong impact on the apparent lacunar pore volumes. ${ }^{71}$

In a previous study by Hesse et al., ${ }^{76}$ five human jaw bone sections (cubes of about $1 \mathrm{~mm}^{3}$ in size) were extracted from three female donors suffering from BRON (Bisphosphonate Related OsteoNecrosis) (aged 43, 63 and 76 years) during the treatments necessary due to the course of the necrosis. Healthy control samples were obtained from debris during wisdom tooth removal from two female donors (aged 25 and 52). ${ }^{76}$ SR- $\mu C T$ measurements were performed in air at the ESRF ID19 with X-ray beam energy $19.1 \mathrm{keV}$. They found that, on average, $50 \%$ and $95 \%$ of the mineralised tissue is located within less than $12 \mu \mathrm{m}$ and $24 \mu \mathrm{m}$ distance of the closest lacuna, respectively. Comparing the average lacunar volume Lc.Vs between the whole samples and the segmented osteonal regions, they revealed that osteonal regions contain significantly smaller lacunae. The lacunar density in these jaw bones ranged from about 16,000 to 50,000 per $\mathrm{mm}^{3}$ and was different to the density reported in femoral sites, probably a consequence of the high bone turnover of the jaw. $^{73}$

The size and shape measurements of the lacunae in jaw bones were found to be in line with those reported for other human bone sites. ${ }^{71,72,74}$ The Lc.Vs decreased with donor age ${ }^{76}$ and the average values of Lc.Vs of BRONJ specimens were within the range of sizes found for the two healthy donors. ${ }^{76}$ The authors presented quantitative $3 \mathrm{D}$ data on how the bone matrix is located in terms 
of distance to the lacunar boundaries. The distances found in human jaw were smaller than those reported for femur. ${ }^{76}$

Hesse et al. ${ }^{77}$ extracted 19 human jaw bone sections (blocks of about $1-3 \mathrm{~mm}^{3}$ in size) from 12 female and 7 male donors (8 female and 2 male donors were suffering from BRONJ). The healthy control samples were from debris obtained during tooth removal. Besides, 7 cadaver specimens originating from the human femoral midshaft and 3 cadaver specimens from the human tibia midshaft of other donors were included in this study. The SR $\mu \mathrm{CT}$ data were obtained at ESRF at beamline ID22NI. The isotropic voxel size was $350 \mathrm{~nm}^{3}$, the energy was set to $16.874 \mathrm{keV}$.

The average lacunar volume ranged from $194 \mathrm{~mm}^{3}$ in the tibia samples to $277 \mathrm{~mm}^{3}$ in the bone jaw. Both median values and variances were significantly higher in the jaw bone compared to the other anatomical sites. The average distance, in which $50 \%$ of the tissue matrix with respect to the closest lacunae was located (Lc.Dist50); was the highest in the femur, followed by the jaw bones and the lowest in the tibiae. ${ }^{77}$

For all investigated sites, the average lacunar density (N.LC/BV) was found to be larger than or equal to $20,000 \mathrm{~mm}^{-3}$. Variations between the anatomical sites reflected those observed for Lc.Dist50, i.e. the lacunar density was the highest in the tibia and the lowest in the femur. The lacunar density in the jaw bones was between those of the other two sites.

Significantly lowest lacunar densities $(p<0.028)$ were observed in the BRONJ samples group. Pronounced differences were observed between jaw bones in comparison to the two peripheral skeletal bone sites. This may be explained by the different embryologic origin of the cells and the different remodelling rates in those tissues, or the different mechanical environment. ${ }^{77}$

On average, the samples treated with bisphosphonates had a lacunar density $14.8 \%$ lower than the healthy jaw bone controls, but still 15\% higher than the femur samples. ${ }^{77}$

\section{Osteogenesis imperfecta}

Albert et al. ${ }^{78}$ have described the effects of the Osteogenesis Imperfecta (OI) on osteocyte lacunae density modulation. Twelve cortical bone specimens were collected from long bone diaphysis of nine children with OI. They showed that average osteocyte lacunar density was $35,661 \mathrm{~mm}^{-3}$ (standard deviation $13,118 \mathrm{~mm}^{-3}$ ). Finally, vascular porosity, osteocyte lacunar density and volumetric tissue mineral density were not significantly associated with donor age. Moreover, the bone material properties were not associated significantly with osteocyte lacunar density or volumetric tissue mineral density. ${ }^{78}$

Although, no significant association between lacunar density and age in the current paediatric OI population was found. These results are within the range previously observed in young adult bone (e.g., 20,000-90,000 lacunae $\left./ \mathrm{mm}^{3}\right) .69,71,72$

The role of osteocyte lacunar properties in bone adaptation and disease is not still understood. Indeed, Mullender et al. ${ }^{79}$ showed a decrease in areal lacunar density for osteoporotic females compared to adult controls, when McCreadie and Hollister ${ }^{80}$ found no significant difference in lacunar size or shape compared to controls.

\section{Conventional synchrotron-based CT applied to rat bone samples}

Healthy rat bone samples

Wittig et al. aimed to link the evolution of bone with age across length scales ranging from the material to the organ level to test whether there is a coupled age-dependence of the osteocyte lacunar geometry or number and, e.g., the quality and mechanical properties of the whole bone in healthy female Wistar rats. ${ }^{81}$

In their work, 42 animals were sacrificed 0, 4, 8, 12, 16 or 28 weeks after study start (ages from 14 to 42 weeks). The global average value of lacunar volume taken over all individuals and age groups is $273 \pm 29 \mu \mathrm{m}^{3}$.

SR $\mu \mathrm{CT}$ data at the mid-diaphysis of the femora was acquired at the beamline for TOmographic Microscopy and Coherent rAdiology experimenTs (TOMCAT) at the Swiss Light Source. X-ray energy used was $18.0 \mathrm{keV}$ and the voxel size of $0.325 \times 0.325 \times 0.325 \mu^{3}$.

For the lacunar stretch Lc.St distribution and average, the age groups did not differ from each other. The lacunar oblateness Lc.Ob distribution and average revealed no significant differences between the age groups. The orientation Lc.Or with respect to the bone long axis, did not reveal differences between age groups. The high resolution studies of osteocyte lacunar geometries showed no significant changes in osteocyte lacunar volume Lc.V, ellipsoidal radii, Lc.St, Lc.Ob or Lc.Or with animal age, despite the changes observed on the organ and tissue level. ${ }^{81}$ 
Pathologic rat bone samples

Bach-Gansmo et al. ${ }^{73}$ investigated whether osteocyte lacunar properties change during immobilisation and subsequent recovery. They used a female Wistar rat cortical bone model with negligible Haversian remodelling effects with temporary immobilisation of one hindlimb induced by botulinum toxin. Their study covered the age span of 14 to 42 weeks of age. After sacrifice, SR $\mu \mathrm{CT}$ data were collected on the proximal half of the femora of the immobilised and the contralateral legs, at the beamline for TOMCAT at the Swiss Light Source (SLS, Paul Scherrer Institute, Villigen, Switzerland) with an X-ray beam energy of $18 \mathrm{keV}$ and an isotropic voxel size of $0.325 \mu \mathrm{m}$. This method was applied to 1.85 million osteocyte lacunae. Their results showed that no significant changes in osteocyte lacunar volume, density, oblateness, stretch or orientation were detected upon immobilisation or subsequent recovery. They concluded that only the bone architecture and not osteocyte lacunar properties dominate the immobilisation response as well as the subsequent recovery. ${ }^{73}$

Previously, Britz et al. also studied the effect of a prolonged unloading in growing rats on cortical osteocyte lacunar density and volume at the distal tibia. ${ }^{82}$

The tibiae from 30-week-old female Sprague-Dawley rats $(n=6)$ that had undergone sciatic neurectomy (immobilised group) at three weeks of age, were compared against the left tibiae from six normal age- and sexmatched rats (control group).

Scanning of the samples was performed at the BioMedical Imaging and Therapy bending magnet beamline (BMITBM; 05B1-1) at the Canadian Light Source. A $23 \mathrm{keV}$ monochromatic beam was employed.

Mean lacunar density was found to be significantly lower $(p<0.05)$ in immobilised bones. Mean lacunar volume was significantly lower for immobilised bones $\left(209 \pm 72 \mu \mathrm{m}^{3}\right)$ compared to control bones $\left.\left(284 \pm 28 \mu^{3}\right)^{3}\right)^{82}$ Their results seem to demonstrate that extreme differences in loading conditions, such as those created by paralysis, do indeed result in changes in osteocyte lacunar density and volume, and so were contradictory with the Bach-Gansmo et al. study. ${ }^{73}$

Tommasini et al. ${ }^{83}$ have quantified 3D changes in cortical osteocyte lacunae and other small porosities induced by estrogen withdrawal and two different osteoporosis treatments in female Sprague-Dawley rats. In their study, six-month old female Sprague-Dawley rats were separated into either age-matched control, untreated ovariectomised (OVX), OVX treated with subcutaneous injections of PTH 1-34 (15 $\mu \mathrm{g} / \mathrm{kg} / \mathrm{d})$, or OVX treated with subcutaneous injections of alendronate (ALN) $(100 \mu \mathrm{g} / \mathrm{kg} / 2 \times / \mathrm{wk})$.

Femoral diaphysis were scanned with SR $\mu \mathrm{CT}$ at Argonne National Laboratory, 2-BM Advanced Photon Source (APS) beamline images were acquired at a photon energy of $20.98 \mathrm{keV}$ with a $750 \mathrm{~nm}$ isotropic voxel size.

Across the overall thickness of the medial cortex, lacunar volume fraction (LC.V/TV) was significantly lower in ALN-treated rats compared to PTH. In the endosteal region, average osteocyte lacunar volume (LC.V) of untreated OVX rats was significantly lower than in agematched controls, indicating a decrease in osteocyte lacunar size in bone formed on the endosteal surface after estrogen withdrawal. The effect of treatments (OVX, ALN, PTH) on the number of lacunae per tissue volume (LC.N/TV) was dependent on the specific location within the cortex (endosteal, intracortical, periosteal). In both the endosteal and intracortical regions, LC.N/TV was significantly lower in ALN than in untreated OVX, suggesting a site-specific effect in osteocyte lacuna density with ALN treatment.

\section{Conventional synchrotron-based CT applied to mice bone samples}

Healthy samples

A few publications have recently characterised osteocyte lacunae and LC.N tomographic parameters in healthy mice. ${ }^{56,84,85}$

Núñez et al..$^{85}$ studied the distribution of the osteocyte lacunae in cortical bone and the influence of age at the tibiofibular junction in 15-week- and 10-monthold female C57BL/6J mice. The tibiae were scanned using SR-CT at the TOMCAT beamline of the SLS with a photon energy of $18.5 \mathrm{keV}$, and a voxel size of $0.65 \mu \mathrm{m}$. They did not observe differences for osteocyte lacunar number density (N.LC/Ct.BV) between both experimental groups. Mean lacunar distance distribution to the nearest bone surface (LC.S.Sp) was significantly higher in the old group versus the young group. Most of the osteocyte lacunae were located within $\sim 40 \mu \mathrm{m}$ from the nearest bone surface in young cortical bone. ${ }^{85}$

Nango et al. ${ }^{56}$ used a Talbot-defocus multiscan tomography using the synchrotron $\mathrm{X}$-ray microscope to study the LCN in mouse tibiae from 12 to 16 -week-old female C57BL/6J mice, at the undulator beamline 20XU in the SR facility Super Photon ring $8 \mathrm{GeV}$ (SPring-8) in Hyogo, 
Japan. Imaging was carried out using monochromatic 9 keV $X$-rays, with a pixel size of $4.34 \mu \mathrm{m}$. For both ages, they have shown that the high mineralisation zone observed near the periosteum and endosteum corresponded to the lamellar zone. The low mineralisation zone located at the centre of the cortical bone corresponded to the non-lamellar (turbulent) zone. Near the endosteum and periosteum in the lamellar zone, lacunae were oriented longitudinally and aligned parallel to the bone surface, and canaliculi ran parallel to each other extending toward or away from the bone surface. In contrast, at the middle of the cortical bone in the turbulent zone, lacunae orientations were irregular. ${ }^{56}$

Matsuo et al. ${ }^{84}$ investigated the non-growth plate type of endochondral ossification of the malleus by SR X-ray CT imaging, with a monochromatic $X$-ray beam $(9.0 \mathrm{keV}$ ) at the beamline BL2OXU of SPring-8 (for Super Photon ring-8 GeV) (Hyogo, Japan), with a voxel size of $0.22 \mu \mathrm{m}$. They assessed the arrangement of osteocytes around capillaries and examined osteocyte lacunae in the malleal processus brevis (mPb) in two adult strain mice (wild type and transgenic mice) which constitutively or inducibly expressed Fosl1 (because of the fos 1 functions in assembly of endothelial cells into capillary tubes). ${ }^{84}$

In both mice strains SR X-ray tomographic microscopy of the isolated malleus enabled them to visualise both capillaries and osteocyte lacunae in reconstructed 3D images. Interestingly, elongated osteocyte lacunae tended to be located along a capillary loop. Furthermore, they detected concentric distribution of osteocyte lacunae around capillary segments.

\section{Mice pathologic samples}

Some publications have described osteocyte lacunae and Lc.N tomographic parameters in different pathologies (OI, osteoporosis, hypercorticism, Sost deficiency, osteopetrosis...). ${ }^{49,56,86-90}$

Carriero et al. ${ }^{86}$ investigated changes in cortical bone tissue porosity of a mouse model of the disease, $\mathrm{OI}$, in comparison to a wild type (WT-C57BL/6). Scanning of tibiae and humeri from wild type mice (C57BL/6), and male OI mice (B6C3Fe a/a-Col1a2 ${ }^{\text {oi/oi }}$ from a maintained local colony), all 8-weeks-old and male were achieved at the beamline for tomographic microscopy and coherent radiology of the SLS, TOMCAT beamline, at a nominal resolution of $700 \mathrm{~nm}$, with a photon energy of $17.5 \mathrm{keV}$.

Carriero et al. ${ }^{86}$ presented morphometric parameters/ measures for the lacunar system, including number of lacunae (N.Lc), lacunar number density (N.Lc/Ct.TV), total lacunar volume (LC.V), lacunar porosity (LC.V/Ct.TV) and mean lacuna volume (bLc.VN = Lc.V/N.Lc). The best-fit ellipsoid gave lacuna major axis (Lc.入1), lacuna interme-

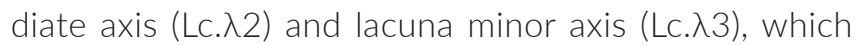
correspond to the lacuna's principal axes. From these values we calculated the mean lacunar equancy (sphericity) (Lc.EqN = Lc. $\lambda 3 / L c . \lambda 1)$, mean lacunar elongation (Lc. $E I=1-L C . \lambda 2 / L c . \lambda 1$ ), mean lacunar flatness (LC.FI = $1-L C$. $\lambda 3 /$ LC. $\lambda 2)$.

The bone matrix of the $\mathrm{Ol}$ bone showed a substantial increase in lacuna number density (N.Lc/Ct.TV, $p<0.001$ ) and lacunar porosity (LC.V/Ct.TV, p<0.001), while the mean lacuna volume (LC.V) was similar in both mouse groups. The lacunar structure and organisation in the Ol cortex differed to that in the WT cortex. In OI bone, osteocyte lacunae were more spherical, but less elongated and flat.

Karunaratne et al. ${ }^{87}$ developed a female mouse model with an $\mathrm{N}$-ethyl-N-nitrosourea-induced corticotrophin releasing hormone promoter mutation $\left(\mathrm{Crh}^{-120 /+}\right)$ that developed hypercorticosteronaemia and osteoporosis. Female $\mathrm{Crh}^{-120 /+}$ mice on a C57BL/6 genetic background (third generation) were used in all experiments; littermate WT mice were used as controls. they were sacrificed at 26 weeks of age. SR $\mu$ CT was performed at the imaging beamline I13-2 at Diamond Light Source (Harwell, UK) to evaluate osteocyte lacunae density at the tibial diaphysis. The effective scanning voxel size was $1.6 \mu^{3}$. Their results indicated a significant reduction in lacunae density $(p<0.001)$ in $\mathrm{Crh}^{-120 /+}$ mice bones. Furthermore, $\mathrm{Crh}^{-120 /+}$ mice bones exhibited some unmineralised tissue.

Nango et al. ${ }^{56}$ analysed bone samples for bone mineral density distribution around the lacuno-canalicular system to assess osteocytic mineral metabolism. To this end, they prepared posterior cortical tibial diaphysis from 7-week-old C57BL/6J mice to fit into a limited view field of synchrotron X-ray tomographic microscopy. Talbotdefocus multiscan $X$-ray microscopy was performed as previously described using a monochromatic light beam of $9 \mathrm{keV}$ at the beamline 20XU at the SR light facility SPring-8 (Hyogo, Japan). Spatial resolution was submicron for defocus phase-contrast tomography. They evaluated the degree of mineralisation around canaliculi in a sagittal section, which vertically spanned the endosteal and periosteal surfaces of tibial cortical bone. Quantitative analysis indicated that mineral density decreased from the periphery to the canaliculus in a pattern reminiscent of a 
$V$-shaped valley. Bone mineral is dissolved around a canaliculus. Seven-week-old C57BL/6J mice were infused with human PTH 1-34 (Peptide Institute, Inc., Osaka, Japan) at $40 \mu \mathrm{g} / \mathrm{kg} /$ day for 6 days.

To validate pericanalicular demineralisation, they analysed tibiae of PTH-infused and control animals, since continuous infusion of PTH-related peptide (PTHrP) induces osteocytic remodelling particularly in trabecular bone. On tibial coronal sections, PTH-infused mice showed more prominent pericanalicular mineral dissoIution than did controls. To determine whether osteoclasts are required for pericanalicular dissolution, they examined mineral density distribution around canaliculi in osteopetrotic mice, which are devoid of osteoclasts. To do so, they used mutant mice lacking either the transcription factor c-Fos (Fos ${ }^{-/-}$mice) or RANKL (Tnfsf11 $1^{-/-}$ mice), both of which exhibit osteopetrotic phenotypes. In samples from Fos ${ }^{-1-}$ mice, they observed low mineral density areas along canaliculi in both coronal and sagittal sections. Tnfsf $11^{-/-}$mice (mice lacking RANKL) also showed pericanalicular demineralisation.

Mader et al. ${ }^{89}$ introduced a framework for the quantitative characterisation of millions of osteocyte lacunae and their spatial relationships in 3D. Indeed, they presented the proposed lacunar descriptors for femoral bones extracted from bones of four different mouse strains. The four mouse strains were namely C57BL/6J (B6) and $\mathrm{C} 3 \mathrm{H} / \mathrm{HeJ}(\mathrm{C} 3 \mathrm{H})$ with a mutation in the growth hormone releasing hormone receptor (Ghrhr, allele symbol $=$ lit): C57BL/6J-Ghrhrlit/J (B6-lit/+), C57BL/6J-Ghrhrlit/ Ghrhrlit (B6-lit/lit), C3H/HeJ-Ghrhrlit/J (C3H-lit/+) and $\mathrm{C} 3 \mathrm{H} / \mathrm{HeJ}-G$ hrhrlit/Ghrhrlit (C3H-lit/lit). They employed SR-CT at the TOMCAT beamline of the SLS to image the mid-diaphysis. The data were acquired and reconstructed at a $1.4 \mu \mathrm{m}$ isotropic voxel size, at an X-ray energy of $17.5 \mathrm{keV}$. Mader et al. ${ }^{89}$ have shown that because in the case of $\mathrm{C} 3 \mathrm{H}$, the growth hormone had a strong effect on the distribution of the osteocyte lacuna. The Lc.N parameter was significantly higher in the $\mathrm{C} 3 \mathrm{Hlit} /+$ group according to the $\mathrm{C} 3 \mathrm{H}$ lit/lit group (respectively 53,287 lacunae vs $33,799, p=0.01$ ). The lacuna lengths and consequently volume were the highest in $\mathrm{C} 3 \mathrm{H}$. The Lc.V $\left(\mu \mathrm{m}^{3}\right)$ was significantly higher in C3Hlit/+ group versus the $\mathrm{C} 3 \mathrm{H}$ lit/lit (577 vs 378, $\mathrm{p}<0.01$ ).

Schneider et al. ${ }^{49}$ have evaluated different 3D quantitative morphometric osteocyte lacunar indices such as number of lacunae (N.LC), lacuna number density (N.LC/ Ct.TV), lacuna volume (LC.V) and lacuna volume density
(LC.V/Ct.TV), in two inbred strains of mice by SR $\mu$ - and nano-CT, at the femoral mid-diaphysis, with nominal resolutions ranging from $3.5 \mathrm{~nm}$ to $700 \mathrm{~nm}$.

To partition bone quality into its regulatory pathways, they chose a mouse model called little (C57BL/6JGhrhrit/J), wherein regulation of bone morphometry is independent of growth hormone and insulin-like growth factor-I (IGF-I) by a mutation. The congenic strain C3.B6Ghrhrlit/J (C3.B6-lit/lit) was created by backcrossing the little mutation from the original $\mathrm{B} 6$ background to $\mathrm{C} 3 \mathrm{H}$. The result of introgressing the little mutation onto the $\mathrm{C} 3 \mathrm{H}$ background is that C3.B6-lit/lit mice have higher bone mineral density (BMD) than B6-lit/lit. Schneider et al. ${ }^{49}$ have shown that in C3.B6-lit/lit, the mean lacuna volume Lc.V was larger than in B6-lit/lit. On the other hand, lacuna number density (N.LC/Ct.TV) was proportionally smaller in C3.B6-lit/lit, so that there is more bone matrix per osteocyte lacuna compared with B6-lit/lit mice. The lacuna volume density (Lc.V/Ct.TV) was constant at $1.3 \%$ for both mouse strains.

Gerbaix et al. ${ }^{90}$ have recently exposed to one-month spaceflight on board the Russian Bion-M1 satellite two groups of five C57BL/6N male mice. The weightless environment during spaceflight induces site-specific bone loss. The 30-day Bion-M1 mission offered a unique opportunity to characterise the skeletal changes after spaceflight and an 8-day recovery period in mature male C57/BL6 mice. To evaluate the impact of spacecraft housing, two groups of ground control mice were investigated. A ground habitat control group (Habitat Control) was placed in the same module under the same climate and food conditions as in the spacecraft, and a standard control group was placed in standard housing conditions (Control). Five cortical femur sections per group were scanned using SR $\mu \mathrm{CT}$ on beamline ID19 at ESRF with a $0.7 \mu \mathrm{m}$ isotopic voxel size, at a fixed energy of $26 \mathrm{keV}$. The total lacunar volume (LC.V) was measured directly by summing the individual volume of each lacuna. The lacunar number density (N.LC/TV) and the lacunar volume density Lc.V/TV (\%) were also assessed. This method allowed for the extraction of the 3D descriptor of lacunae: length ( $\left(L C . L_{1}\right)$, width (LC. $\left.L_{2}\right)$ and depth $\left(L C . L_{3}\right)$. The shape of the lacunae was evaluated by the ratios of the axis lengths $L c . L_{1} / L c . L_{2}$ and $L c . L_{1} / L c . L_{3}$. They observed that in the flight animal group, the osteocyte lacunae displayed a reduced volume and a more spherical shape, and empty lacunae were highly increased (+344\% versus Habitat Control). 
Mosey et al. ${ }^{88}$ hypothesised that Sost deficiency results in altered osteoblast-to-osteocyte transition and vascular network formation. They imaged tibiae from Sost $\mathrm{KO}$ mice and their WT counterparts with high-resolution CT to examine whether lack of sclerostin influences the morphometric properties of lacunae and vascular canal porosity relating to osteocytes and vessels.

Male Sost $\mathrm{KO}$ and WT mice ( $n=6 /$ group) were sacrificed at 12 weeks of age. Samples were imaged by using benchtop and synchrotron nano-computed tomography at the tibiofibular junction. Tibiofibular junctions were scanned using SR-CT at the TOMCAT beamline of the SLS at a voxel size of $0.65 \mu \mathrm{m}$. The photon energy was $18.5 \mathrm{keV}$.

Mosey et al. ${ }^{88}$ have shown a greatest total number of osteocyte lacunae (N.LC; $p<0.001$ ) in Sost KO compared with WT mice, in images obtained from nano-CT and synchrotron. The average lacunar volume (Lc.Avg.V), total lacunar volume occupied by all lacunar pores (Lc. Tot.V) and lacunar diameter (LC.D) were not significantly different in datasets of either imaging modalities between Sost KO and WT mice, at the tibiofibular junction. They concluded that the significant increases in bone mass induced by Sost deficiency are not accompanied by any significant modification in the density, organisation or shape of osteocyte lacunae.

\section{Ptychographic X-ray CT}

Very few publications have recently characterised osteocyte lacunae and LCN ptychographic parameters in rats. ${ }^{91-93}$ Main parameters used are presented in Table 1.

Kerschnitzki et al. ${ }^{91}$ visualised and topologically quantified the osteocyte network in mineralised bone sections with CLSM. At the same region of the sample, synchrotron small-angle $\mathrm{X}$-ray scattering was used to determine nanoscopic bone mineral particle size and arrangement relative to the cell network. To do that, native ovine fibrolamellar (plexiform) bone samples were collected from the midshaft region of the femur of a mature 5-yearold sheep. Bone samples were scanned at the Nanofocus beamline ID13 at ESRF using a monochromatic 14-keV $X$-ray beam with a beam size of $500 \mathrm{~nm}$.

They demonstrated that the distribution of the shortest distance of a network voxel to its adjacent osteocyte showed that almost $70 \%$ of the canalicular network is within a transport distance of only $10 \mu \mathrm{m}$. The mean canalicular length (travel distance) between the nodes was about $2.15 \mu \mathrm{m}$. The processed osteocyte network data also contained quantitative information about the amount of mineral residing in the vicinity of the osteocyte network. They found that within a distance as small as $1 \mu \mathrm{m}, 60 \%$ of the mineralised matrix were located, and within $1.4 \mu \mathrm{m}, 80 \%$ of the mineralised matrix were located.

Ciani et al. ${ }^{92,93}$ applied SR ptychographic X-ray CT to characterise the lacunae and LCN in a male Wistar rat model of glucocorticoid-induced osteoporosis (GIO). After acclimation, at 19 weeks of age, the rats were randomly assigned to a control group (SHAM) or a methylprednisolone-treated group (GIO). Methylprednisolone (Sigma, France) was injected subcutaneously at $5 \mathrm{mg} \mathrm{kg}^{-1}$, five times per week. The control rats were subcutaneously injected with vehicle (saline) twice a week. The protocol lasted nine weeks. Transverse sections from upper half of the tibial diaphysis, approximately $1 \mathrm{~cm}$-diameter, were sliced using a rotary diamond saw (Dremel 300), then manually sectioned to $\sim 50 \times 50 \times 150 \mu^{3}$ and mounted on needles. For ptychography $X$-ray CT image acquisition, a total of 11 such free-standing bone specimens were analysed by this method; 6 samples from the GIO group belonging to 4 different rats, and 5 samples from the SHAM group, belonging to 5 different rats.

The samples were imaged at the cSAXS beamline of the SLS in three different rounds of experiments. The X-ray photon energy, E, was $6.2 \mathrm{keV}$ in all cases. The voxel side length belongs to the interval (39-44) nm (Figure 2). The measured parameters were as follows.

The volume of each lacuna was calculated by two methods. LcV1: number of empty voxels excluding canaliculi, LcV2: volume lacuna obtained by a fitting procedure. The optimised fit residual, Lc. Fopt is the root-meansquare (rms) difference between the best-fit ellipsoid and the lacuna surface, ${ }^{92}$ which gives a measure of the surface irregularity of the lacuna, Ca.Dm = canaliculi diameter, Ca.V/MV = microporosity as the ratio of the volume occupied by canaliculi to the total matrix tissue volume; $\mathrm{Ca} . \mathrm{Nb}=$ number of canaliculi originating from the lacuna per ellipsoidal surface unit; Ca.Pr = mean number of primary canaliculi directly connected to the lacuna; Ca.Db = length of the first dendritic branching distance.

The ptychographic images revealed significant differences in the number of canaliculi originating from the lacuna per ellipsoidal surface unit, Ca.Nb ( $p=0.0106)$, and the 3D morphology of the lacuna $(p=0.0064)$, between 
GIO and SHAM groups. Moreover, the mean canalicular diameter, Ca.Dm, was slightly statistically non-significantly smaller in GIO $(152 \pm 6.5) \mathrm{nm}$ than in the SHAM group $(165 \pm 8) \mathrm{nm}(\mathrm{p}=0.053)$.

\section{Synchrotron deep ultraviolet fluorescence spectroscopic microscopy}

Concerning the DUV micro-spectroscopy, this technique is used for investigating autofluorescent components in bone tissues, especially to analyse the osteocyte composition and the surrounding matrix. Pallu et al. ${ }^{62}$ have observed three fluorescents peaks corresponding to tyrosine $(305 \mathrm{~nm})$, tryptophan $(333 \mathrm{~nm})$ and collagen $(385 \mathrm{~nm})$ and two ratios, tyrosine/collagen ratio and tryptophan/ collagen ratio, to analyse the effects of alcoholism in a rat model (Figure 3). They have shown that tyrosine/collagen and tryptophan/collagen ratios were higher in the strong alcohol consumption group according to control rats. Tryptophan might be related to the serotonin metabolism involved in bone formation, while tyrosine is involved in the activity of tyrosine kinases and phosphatases in osteocytes, translating a change in bone remodelling. ${ }^{62,94}$ Among the two doses of alcohol used in Pallu et al., ${ }^{67}$ the lower dose (A25 \% v/v) induced a statistically significant difference for the tyrosine/collagen ratio (osteocyte vs matrix: $0.96 \pm 0.10$ vs $1.11 \pm 0.12, p=0.0025)$ and for the tryptophan/collagen ratio (osteocyte vs matrix: $1.89 \pm 0.18$ vs $2.24 \pm 0.20, p=0.0001)$. Main parameters used are presented in Table 1.

Pallu et al., ${ }^{62,67}$ have clearly demonstrated that tyrosine/ collagen and tryptophan/collagen ratios were higher in the strong alcohol consumption group. Tryptophan might be related to the serotonin or 1-25 dihydroxyvitamin $D$ metabolism involved in bone formation, while tyrosine is involved in the activity of tyrosine kinases and phosphatases in osteocytes.

To provide quantitative biochemical targets in the osteocyte lacuna and its surrounding matrix in bone healthy and osteoporotic samples, imaging at the micron scale gives innovative information on the role of biological targets (such as amino acids) in the bone remodelling process; through the Proteins Tyrosine Kinases (PTK)/ Proteins Tyrosine Phosphatases balance, or through other signalling transduction pathways involved in the bone metabolism such as serotonin, 1-25 dihydroxyvitamin D, osteostatin. ${ }^{62}$ These metabolic pathways might be involved in the deleterious effects of the osteoporosis and could contribute to the identification of new therapeutic targets for this pathology, as well as for other osteo-articular diseases.

The use of synchrotron light as a source of $U V$ permitted the excitation light to be tuned to correspond with the absorption of endogenous fluorochromes (NADH, collagen, tyrosine, tryptophan, lipo-pigments, elastin, pyridoxins). Since the DUV has short wavelengths, its best resolution is around $120 \mathrm{~nm}$. Further, as for FT-IR micro-spectroscopy, this method is not precise enough to characterise quantitative amounts of the different biochemical targets at the canaliculi scale.

\section{Synchrotron FT-IR spectroscopic imaging}

Bone components were also measured using synchrotron FTIRM. As previously explained, FTIRM is the fluorescence-assisted synchrotron Fourier transform infrared micro-spectroscopy. FTIRM is usually used to investigate the structural features of bone organic molecules by examining the frequency at which molecular bonds vibrate (stretching and bending).

The phosphates $\left(650-500 \mathrm{~cm}^{-1}\right)$, carbonates (905$\left.825 \mathrm{~cm}^{-1}\right)$, proteins (amide I) $\left(1668-1623 \mathrm{~cm}^{-1}\right)$ and collagen cross-links are obtained.

IR spectral data are presented as ratios of phosphate/ protein (overall matrix mineralisation) and carbonate/ protein (substitution of carbonate into hydroxyapatite). The carbonate/phosphate ratio is used to judge phosphate substitution by carbonate and indicates the degree of mineralisation. The collagen cross-linking is determined using FT-IR micro-spectroscopy, it is the $1660 / 1690 \mathrm{~cm}^{-1}$ ratio which corresponds to the ratio of non-reducible to reducible collagen cross-links in bone. This non-reducible cross-link/reducible cross-link ratio is an indicator of maturity of collagen. Mineral features such crystallite and perfection (crystallinity) are also obtained using synchrotron FTIRM.

These ratios are used in different models (mouse, rabbit, rat, monkey...), tissues (osteonal vs insterstitial bone or cortical vs trabecular bone), age or pathology (osteoporosis, fibrous dysplasia). To analyse the bone mineralisation process in healthy subjects, bone components and mineralisation were obtained in young mice aged 1 to 40 days, compared to adult (450 days). ${ }^{64}$ The degree of mineralisation (phosphate/protein ratio) and the mineral crystallinity increase with age of mice without carbonate substitution and collagen crosslinking changes. The progression of mineralisation in this osteonal bone has also been observed in young rabbits 
by Fuchs et al. ${ }^{95}$ rapidly in the first 18 days, then slower until it was stabilised around 350 days. An early postovariectomy model of osteoporosis was proposed by Huang et al. ${ }^{96}$ They demonstrated that new cortical bone formed in ovariectomised monkeys had a higher phosphate content, a lower carbonate content and more mature collagen cross-links compared to the SHAMoperated group. In contrast, trabecular bone had no change in phosphate content, a lower carbonate and immature cross-linking. These results suggest that during remodelling after ovariectomy, the quality and quantity of newly formed tissue is affected. Kazakia et al. ${ }^{97}$ proposed a model mimicking the effects of fibrous dysplasia of the bone in transgenic mice. They have observed a lower mineral/matrix ratio, a higher carbonate/phosphate ratio, a lower crystallinity and a lower cross-link ratio in transgenic mice compared to wild type at 6 or 15 weeks. They concluded that activation of osteoblasts leads to deposition of quantities of immature bone with reduced mineralisation.

The common point of all these studies is to understand the mineralisation and matrix composition of bone tissue mainly at the osteon level. A study looking at a lower scale in cortical bone in an alcohol-induced osteoporosis model, described the relationship between osteocyte lacuna and surrounding matrix for this chemical point of view ${ }^{67}$ (Figure 4). The main parameters used are presented in Table 1.
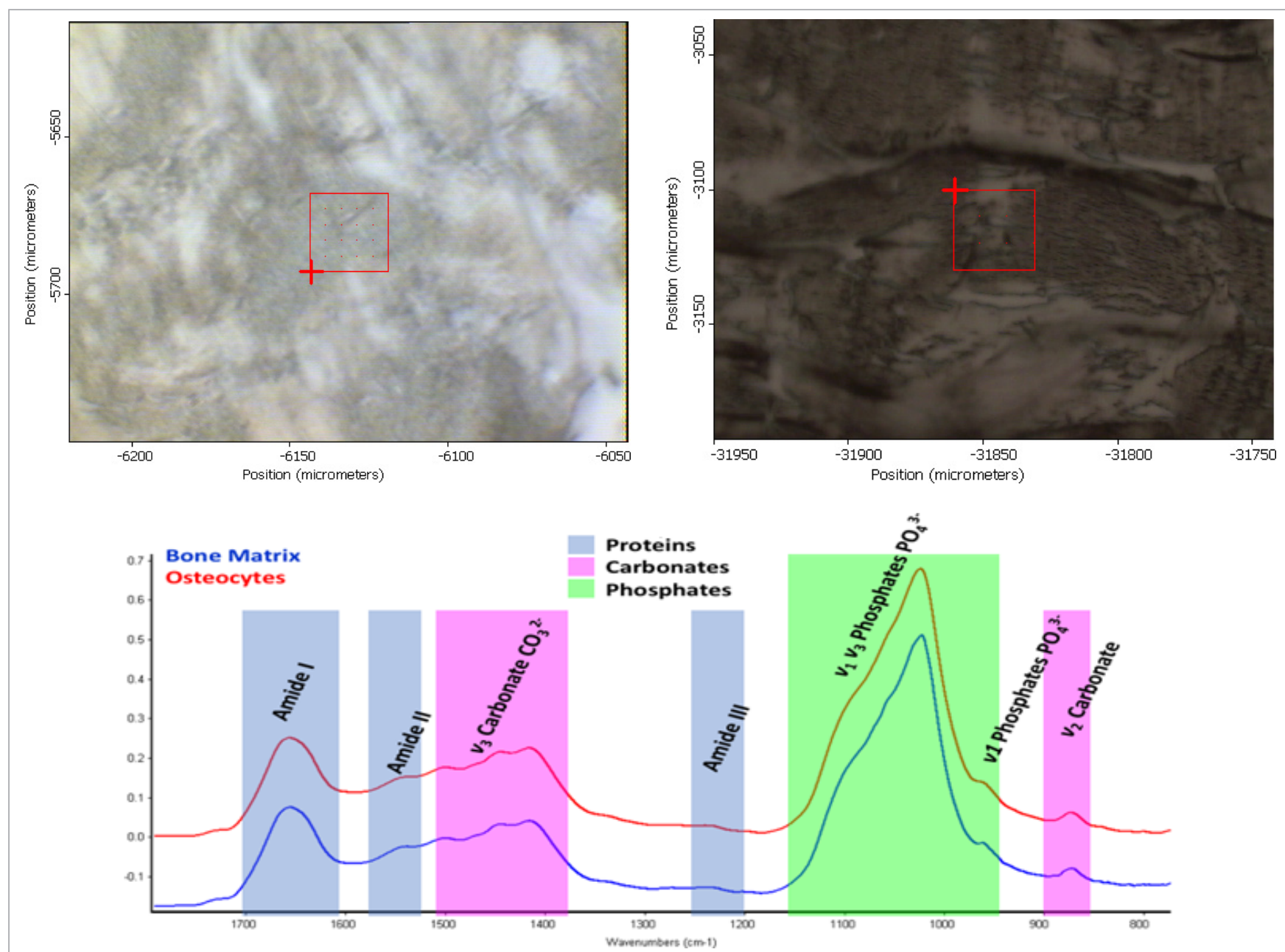

Figure 4. ROI from transmission microscopy acquisition in osteocyte lacunae and surrounding matrix ROI. Acquisitions were performed at the SMIS beamline, Synchrotron SOLEIL, at the spectral resolution of $4 \mathrm{~cm}^{-1}$. A: ROI from transmission microscopy acquisition in control Wistar male rats (acquisition from the upper half of the cortical tibial diaphysis). B: ROI from transmission microscopy acquisition in alcohol-induced osteoporotic Wistar male rats (acquisition from the upper half of the cortical tibial diaphysis) (PhD of Delphine Maurel, Université d'Orléans, France). C: FT-IR spectra acquired in osteocyte lacuna and its surrounding matrix. 


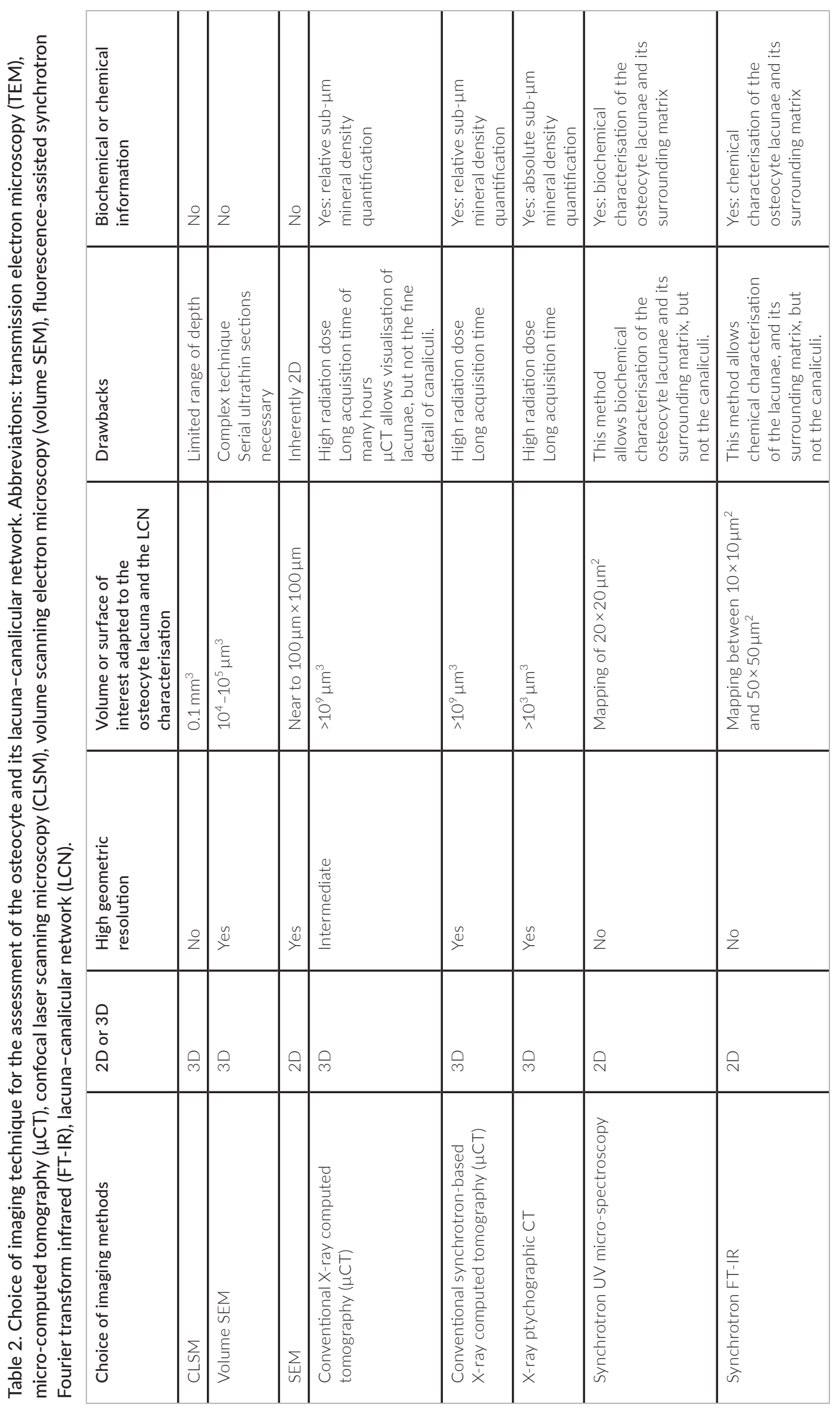


FT-IR microscopy and spectroscopy on the SMIS beamline at the Synchrotron SOLEIL helps to evaluate collagen mature/immature ratios, Phosphates/Amide I, Carbonates/Amide I ratios, crystallinity ratios both in the osteocyte lacuna and in its surrounding matrix. Synchrotron light is 1000 times brighter than conventional IR light sources, which improves the spatial resolution and signal-to-noise ratio of the micro-spectrometer, allowing data to be acquired at the diffraction limit.

This methodology at a such resolution is not sufficiently precise to characterise quantitative amounts of the different chemical ratios at the canaliculi level, but can discriminate between the osteocyte lacunae and their surrounding matrix. These data could be collected both in control condition, and the osteoporotic experimental context, and could also constitute an interesting target for a better understanding of the effect of the pathology or its treatments at the micron scale.

\section{Conclusion}

What is the best choice of imaging techniques for the assessment of the osteocyte in its lacuna and its lacuna-canalicular network among the different 2D and 3D methods? This choice needs to evaluate the most adequate bone surface or volume of interest, the degree of difficulty to prepare the samples, the resolution, the duration of the acquisitions, the nature of the information obtained (geometric, chemical, biochemical data), the capacity to bring information on both osteocyte lacuna and its LCN or not, among the different methods. A synthesis on these different technics is presented in Table 2.

Among the 2D imaging methods, in terms of resolution, the best method remains the TEM method with a resolution $<1 \mathrm{~nm}$. Meanwhile, this method has many limitations, such as requiring cryofixation of ultrathin bone sections, complex sample preparation and a limited field of view. The second-best method is the $S E M$ with a resolution of $3 \mathrm{~nm}$. These two methods bring ultrastructural 2D parameters on osteocyte lacunae and canaliculi (Table 2).

Among the 3D imaging methods, in terms of resolution, the best methods to characterise both the osteocyte lacuna and its LCN remain ptychographic $X$-ray CT with a highest resolution of $16 \mathrm{~nm}^{98}$ and volume SEM with a $x / y$-resolution of $<5 \mathrm{~nm}$ and a z-resolution near to $10-15 \mathrm{~nm}$. However, ptychographic X-ray CT's main limitation is the long acquisition time per bone sample (Table 2). Volume SEM's main limitation is the fact that it is a complex method and it needs bone serial ultrathin sections.

In synchrotron DUV and FT-IR micro-spectroscopy, the resolution used is only adapted to, respectively, discriminate between biochemical and chemical composition of the osteocyte lacuna versus its surrounding matrix. Both methods do not have enough resolution to characterise the LCN.

\section{Acknowledgements}

The authors acknowledge Paul Scherrer Institut, Villigen, Switzerland, for provision of synchrotron radiation beam time at the CSAXS beamline of the SLS (proposals 20110196, 20120152) and the Synchrotron SOLEIL, Gif-sur-Yvette, France, for provision of synchrotron radiation beam time at the DISCO and SMIS beamlines (Synchrotron SOLEIL proposal 20090113). The authors further acknowledge Professor Paul Dumas, Dr Christophe Sandt and Dr Matthieu Réfregiers for their assistance in the imaging experiments at the Synchrotron SOLEIL.

\section{Table of abbreviations}

FT-IR: Fourier transform infrared

LCN: lacuno-canalicular network

CD 44: cluster differentiation 44

DMP1: dentin matrix protein 1

MEPE: matrix extracellular phosphoglycoprotein

IGF: insulin-like growth factor

NO: nitric oxide

FGF: fibroblast growth factor

LM: light microscopy

CLSM: confocal laser scanning microscopy

EM: electron microscopy

TEM: transmission electron microscopy

UHVEM: ultra-high voltage electron microscopy

SEM: scanning electron microscopy

SHG: second harmonic generation

FIB: focused ion beam

SBF: serial block face

$\mu \mathrm{CT}$ : micro-computed tomography 
DUV: deep ultraviolet

FTIRM: Fourier transform infrared micro-spectroscopy

HPLC: high-performance liquid chromatography

BV/TV: bone volume/tissue volume

Lc.L1: length of the axis 1 of the lacuna

Lc.L2: length of the axis 2 of the lacuna

Lc.V: mean volume of the lacuna

BRONJ: bisphosphonate-related osteonecrosis of the jaw

PNCT: phase nano-computed tomography

ESRF: European Synchrotron Radiation Facility

BRON: bisphosphonate related osteonecrosis

SR: synchrotron radiation

Lc.Dist50: distance within which $50 \%$ of the mineralised bone tissue is located with respect to the closest lacunar surface.

N.Lc/BV: average lacunar density

OI: Osteogenesis Imperfecta

TOMCAT: TOmographic Microscopy and Coherent rAdiology experimenTs

Lc.St: lacunar stretch

Lc.Ob: lacunar oblateness

Lc.Or: lacunar orientation

BMITBM: BioMedical Imaging and Therapy bending magnet beamline

OVX: Ovariectomised

PTH: parathyroid hormone

ALN: alendronate

APS: Advanced Photon Source

Lc.V/TV: lacunar volume fraction

Lc.N/TV: number of lacunae per tissue volume

N.Lc/Ct.BV or N.Lc/Ct.TV: osteocyte lacunar number density

Lc.S.Sp: mean lacunar distance distribution to the nearest bone surface

$\mathrm{mPb}$ : malleal processus brevis

WT: Wild Type

Lc.V/Ct.TV: lacunar porosity

SLS: Swiss Light Source

N.Lc: number of lacunae

Lc.Avg.V: average lacunar volume

Lc.Tot.V: total lacunar volume occupied by all lacunar pores

Lc.D: lacunar diameter

GIO: glucocorticoid-induced osteoporosis

LcV1: number of empty voxels corresponding to the volume of each lacuna excluding canaliculi

LcV2: volume lacuna obtained by a fitting procedure
Lc.Fopt: root-mean-square difference between the best-fit ellipsoid and the lacuna surface

Ca.Dist ${ }_{50}$ : distance to canaliculi boundary in which 50\% of the BV is located

Ca.Dm: canaliculi diameter

Ca.V/MV: microporosity as the ratio of the volume occupied by canaliculi to the total matrix tissue volume

Ca.Nb: number of canaliculi originating from the lacuna per ellipsoidal surface unit

Ca.Pr: mean number of primary canaliculi directly connected to the lacuna

$\mathrm{Ca}$.Db: length of the first dendritic branching distance

Ca.S/BV: surface of the canaliculi according to the bone volume

2PF: two-photon excitation fluorescence

\section{References}

1. L.F. Bonewald, "Osteocytes as dynamic multifunctional cells", Ann. N. Y. Acad. Sci. 1116(1), 281 (2007). https://doi.org/10.1196/annals.1402.018

2. A. Vatsa, R.G. Breuls, C.M. Semeins, P.L. Salmon, T.H. Smit and J. Klein-Nulend, "Osteocyte morphology in fibula and calvaria - is there a role for mechanosensing?", Bone 43(3), 452 (2008). https://doi. org/10.1016/j.bone.2008.01.030

3. S.C. Manolagas, "Choreography from the tomb: An emerging role of dying osteocytes in the purposeful, and perhaps not so purposeful, targeting of bone remodeling", BoneKEy Knowledge Environment 3(1), 5 (2006). http://triggered. stanford.clockss.org/ServeContent?url=https \%3A\%2F\%2Fknowledgeenvironment.stanford. clockss.org\%2F2006\%2Fbonekey_2006. bonekey20060193_xml_pdf\%2Fbonekey20060193. html

4. C. Palumbo, S. Palazzini, D. Zaffe and G. Marotti, "Osteocyte differentiation in the tibia of newborn rabbit: an ultrastructural study of the formation of cytoplasmic processes", Acta Anat. (Basel) 137(4), 350 (1990). https://doi.org/10.1159/000146907

5. J.E. Aubin and K. Turksen, "Monoclonal antibodies as tools for studying the osteoblast lineage", Microsc. Res. Tech. 33(2), 128 (1996). https://doi.org/10.1002/ (SICI)1097-0029(19960201)33:2<128::AIDJEMT4>3.0.CO;2-P 
6. C.A. Baud, "Submicroscopic structure and functional aspects of the osteocyte", Clin. Orthop. Relat. Res. 56(56), 227 (1968). https://doi. org/10.1097/00003086-196801000-00025

7. T.A. Franz-Odendaal, B.K. Hall and P.E. Witten, "Buried alive: how osteoblasts become osteocytes”, Dev. Dyn. 235(1), 176 (2006). https://doi. org/10.1002/dvdy.20603

8. D.A. Cameron, H.A. Paschall and R.A. Robinson, "Changes in the fine structure of bone cells after the administration of parathyroid extract", J. Cell Biol. 33(1), 1 (1967). https://doi.org/10.1083/jcb.33.1.1

9. S. Weinbaum, S.C. Cowin and Y. Zeng, "A model for the excitation of osteocytes by mechanical loadinginduced bone fluid shear stresses", J. Biomech.

27(3), 339 (1994). https://doi.org/10.1016/00219290(94)90010-8

10. S.C. Cowin, "Mechanosensation and fluid transport in living bone", J. Musculoskelet. Neuronal Interact. 2(3), 256 (2002). https://www.ncbi.nlm.nih.gov/pub$\mathrm{med} / 15758447$

11. Y. Han, S.C. Cowin, M.B. Schaffler and S. Weinbaum, "Mechanotransduction and strain amplification in osteocyte cell processes", Proc. Natl. Acad. Sci. USA 101(47), 16689 (2004). https://doi.org/10.1073/ pnas.0407429101

12. R.E. Uzbekov, D.B. Maurel, P.C. Aveline, S. Pallu, C.L. Benhamou and G.Y. Rochefort, "Centrosome fine ultrastructure of the osteocyte mechanosensitive primary cilium", Microsc. Microanal. 18(6), 1430 (2012). https://doi.org/10.1017/ S1431927612013281

13. A. Kawata and Y. Mikuni-Takagaki, "Mechanotransduction in stretched osteocytestemporal expression of immediate early and other genes", Biochem. Biophys. Res. Commun. 246(2), 404 (1998). https://doi.org/10.1006/bbrc.1998.8632

14. A.G. Robling, P.J. Niziolek, L.A. Baldridge, K.W. Condon, M.R. Allen, I. Alam, S.M. Mantila, J. GluhakHeinrich, T.M. Bellido, S.E. Harris and C.H. Turner, "Mechanical stimulation of bone in vivo reduces osteocyte expression of Sost/sclerostin", J. Biol. Chem. 283(9), 5866 (2008). https://doi.org/10.1074/ jbc.M705092200

15. S.C. Rawlinson, A.J. el-Haj, S.L. Minter, I.A. Tavares, A. Bennett and L.E. Lanyon, "Loading-related increases in prostaglandin production in cores of adult canine cancellous bone in vitro: a role for prostacyclin in adaptive bone remodeling?", J. Bone Miner. Res. 6(12), 1345 (1991). https://doi. org/10.1002/jbmr.5650061212

16. N. Basso and J.N.M. Heersche, "Effects of hind limb unloading and reloading on nitric oxide synthase expression and apoptosis of osteocytes and chondrocytes", Bone 39(4), 807 (2006). https://doi. org/10.1016/j.bone.2006.04.014

17. B.S. Noble and J. Reeve, "Osteocyte function, osteocyte death and bone fracture resistance", Mol. Cell. Endocrinol. 159(1-2), 7 (2000). https://doi. org/10.1016/S0303-7207(99)00174-4

18. I. Urakawa, Y. Yamazaki, T. Shimada, K. lijima, H. Hasegawa, K. Okawa, T. Fujita, S. Fukumoto and T. Yamashita, "Klotho converts canonical FGF receptor into a specific receptor for FGF23", Nature 444(7120), 770 (2006). https://doi.org/10.1038/ nature05315

19. I.Z. Ben-Dov, H. Galitzer, V. Lavi-Moshayoff, R. Goetz, M. Kuro-o, M. Mohammadi, R. Sirkis, T. Naveh-Many and J. Silver, "The parathyroid is a target organ for FGF23 in rats", J. Clin. Invest. 117(12), 4003 (2007). https://doi.org/10.1172/JCl32409

20. F.M. Hannan, N.A. Athanasou, J. Teh, C.L.M.H. Gibbons, B. Shine and R.V. Thakker, "Oncogenic hypophosphataemic osteomalacia: biomarker roles of fibroblast growth factor 23, 1,25-dihydroxyvitamin D3 and lymphatic vessel endothelial hyaluronan receptor 1", Eur. J. Endocrinol. 158(2), 265 (2008). https://doi.org/10.1530/EJE-07-0485

21. G. Marotti, M. Ferretti, F. Remaggi and C. Palumbo, "Quantitative evaluation on osteocyte canalicular density in human secondary osteons", Bone 16(1), 125 (1995). https://doi. org/10.1016/8756-3282(95)80022-I

22. F. Remaggi, V. Canè, C. Palumbo and M. Ferretti, "Histomorphometric study on the osteocyte lacunocanalicular network in animals of different species. I. Woven-fibered and parallel-fibered bones", Ital. J. Anat. Embryol. 103(4), 145 (1998).

23. S.W. Hell, "Far-field optical nanoscopy", Science 316(5828), 1153 (2007). https://doi.org/10.1126/ science.1137395

24. P. Schneider, M. Meier, R. Wepf and R. Müller, "Towards quantitative 3D imaging of the osteocyte lacuno-canalicular network", Bone 47(5), 848 (2010). https://doi.org/10.1016/j.bone.2010.07.026 
25. L.-D. You, S. Weinbaum, S.C. Cowin and M.B. Schaffler, "Ultrastructure of the osteocyte process and its pericellular matrix", Anat. Rec. A Discov. Mol. Cell. Evol. Biol. 278(2), 505 (2004). https://doi. org/10.1002/ar.a.20050

26. C.W. Jones, D. Smolinski, A. Keogh, T.B. Kirk and M.H. Zheng, "Confocal laser scanning microscopy in orthopaedic research", Prog. Histochem. Cytochem. 40(1), 1 (2005). https://doi.org/10.1016/j. proghi.2005.02.001

27. H. Kamioka, T. Honjo and T. Takano-Yamamoto, "A three-dimensional distribution of osteocyte processes revealed by the combination of confocal laser scanning microscopy and differential interference contrast microscopy", Bone 28(2), 145 (2001). https://doi.org/10.1016/S8756-3282(00)00421-X

28. Y. Sugawara, R. Ando, H. Kamioka, Y. Ishihara, T. Honjo, N. Kawanabe, H. Kurosaka, T. TakanoYamamoto and T. Yamashiro, "The three-dimensional morphometry and cell-cell communication of the osteocyte network in chick and mouse embryonic calvaria", Calcif. Tissue Int. 88(5), 416 (2011). https:// doi.org/10.1007/s00223-011-9471-7

29. L. Cardoso, S.P. Fritton, G. Gailani, M. Benalla and S.C. Cowin, "Advances in assessment of bone porosity, permeability and interstitial fluid flow", J. Biomech. 46(2), 253 (2013). https://doi. org/10.1016/j.jbiomech.2012.10.025

30. H. Sano, J. Kikuta, M. Furuya, N. Kondo, N. Endo and M. Ishii, "Intravital bone imaging by two-photon excitation microscopy to identify osteocytic osteolysis in vivo", Bone 74, 134 (2015). https://doi. org/10.1016/j.bone.2015.01.013

31. D. Tokarz, R. Cisek, M.N. Wein, R. Turcotte, C. Haase, S.-C.A. Yeh, S. Bharadwaj, A.P. Raphael, H. Paudel, C. Alt, T.-M. Liu, H.M. Kronenberg and C.P. Lin, "Intravital imaging of osteocytes in mouse calvaria using third harmonic generation microscopy", PLoS One 12(10), e0186846 (2017). https://doi. org/10.1371/journal.pone.0186846

32. R. Genthial, M. Gerbaix, D. Farlay, L. Vico, E. Beaurepaire, D. Débarre and A. Gourrier, "Third harmonic generation imaging and analysis of the effect of low gravity on the lacuno-canalicular network of mouse bone", PLoS One 14(1), e0209079 (2019). https://doi.org/10.1371/journal. pone.0209079
33. P. Schneider, M. Meier, R. Wepf and R. Müller, "Serial FIB/SEM imaging for quantitative 3D assessment of the osteocyte lacuno-canalicular network", Bone 49(2), 304 (2011). https://doi.org/10.1016/j. bone.2011.04.005

34. L.M. McNamara, R.J. Majeska, S. Weinbaum, V. Friedrich and M.B. Schaffler, "Attachment of osteocyte cell processes to the bone matrix", Anat. Rec. (Hoboken) 292(3), 355 (2009). https://doi. org/10.1002/ar.20869

35. J.G. White, E. Southgate, J.N. Thomson and S. Brenner, "The structure of the nervous system of the nematode Caenorhabditis elegans", Philos. Trans. R. Soc. Lond. B Biol. Sci. 314(1165), 1 (1986). https://doi. org/10.1098/rstb.1986.0056

36. K.M. Harris, E. Perry, J. Bourne, M. Feinberg, L. Ostroff and J. Hurlburt, "Uniform serial sectioning for transmission electron microscopy", J. Neurosci. 26(47), 12101 (2006). https://doi.org/10.1523/ JNEUROSCI.3994-06.2006

37. L. Bonetta, "Zooming in on electron tomography", Nat. Methods 2(2), 139 (2005). https://doi. org/10.1038/nmeth0205-139

38. R. Mclntosh, D. Nicastro and D. Mastronarde, "New views of cells in 3D: an introduction to electron tomography", Trends Cell Biol. 15(1), 43 (2005). https://doi.org/10.1016/j.tcb.2004.11.009

39. A. Takaoka, T. Hasegawa, K. Yoshida and H. Mori, "Microscopic tomography with ultra-HVEM and applications", Ultramicroscopy 108(3), 230 (2008). https://doi.org/10.1016/j.ultramic.2007.06.008

40. H. Kamioka, S.A. Murshid, Y. Ishihara, N. Kajimura, T. Hasegawa, R. Ando, Y. Sugawara, T. Yamashiro, A. Takaoka and T. Takano-Yamamoto, "A method for observing silver-stained osteocytes in situ in 3-microm sections using ultra-high voltage electron microscopy tomography", Microsc. Microanal. 15(5), 377 (2009). https://doi.org/10.1017/ S1431927609990420

41. H. Kamioka, Y. Kameo, Y. Imai, A.D. Bakker, R.G. Bacabac, N. Yamada, A. Takaoka, T. Yamashiro, T. Adachi and J. Klein-Nulend, "Microscale fluid flow analysis in a human osteocyte canaliculus using a realistic high-resolution image-based three-dimensional model", Integr. Biol. 4(10), 1198 (2012). https:// doi.org/10.1039/c2ib20092a 
42. I. Wacker and R.R. Schroeder, "Array tomography", J. Microsc. 252(2), 93 (2013). https://doi.org/10.1111/ jmi.12087

43. R. Müller, "Hierarchical microimaging of bone structure and function", Nat. Rev. Rheumatol. 5(7), 373 (2009). https://doi.org/10.1038/ nrrheum.2009.107

44. R.P. van Hove, P.A. Nolte, A. Vatsa, C.M. Semeins, P.L. Salmon, T.H. Smit and J. Klein-Nulend, "Osteocyte morphology in human tibiae of different bone pathologies with different bone mineral density-is there a role for mechanosensing?", Bone 45(2), 321 (2009). https://doi.org/10.1016/j. bone.2009.04.238

45. M. Salomé, F. Peyrin, P. Cloetens, C. Odet, A.M. Laval-Jeantet, J. Baruchel and P. Spanne, "A synchrotron radiation microtomography system for the analysis of trabecular bone samples", Med. Phys. 26(10), 2194 (1999). https://doi. org/10.1118/1.598736

46. C. Chappard, S. Bensalah, C. Olivier, P.J. Gouttenoire, A. Marchadier, C. Benhamou and F. Peyrin, "3D characterization of pores in the cortical bone of human femur in the elderly at different locations as determined by synchrotron micro-computed tomography images", Osteoporos. Int. 24(3), 1023 (2013). https://doi.org/10.1007/s00198-012-2044-4

47. C. Chappard, F. Peyrin, A. Bonnassie, G. Lemineur, B. Brunet-Imbault, E. Lespessailles and C.L. Benhamou, "Subchondral bone micro-architectural alterations in osteoarthritis: a synchrotron microcomputed tomography study", Osteoarthritis Cartilage 14(3), 215 (2006). https://doi.org/10.1016/j. joca.2005.09.008

48. F. Peyrin, M. Salome, P. Cloetens, A.M. Laval-Jeantet, E. Ritman and P. Rüegsegger, "Micro-CT examinations of trabecular bone samples at different resolutions: 14, 7 and 2 micron level", Technol. Health Care 6(5-6), 391 (1998). https://doi.org/10.3233/THC1998-65-611

49. P. Schneider, M. Stauber, R. Voide, M. Stampanoni, L.R. Donahue and R. Müller, "Ultrastructural properties in cortical bone vary greatly in two inbred strains of mice as assessed by synchrotron light based micro- and nano-CT", J. Bone Miner. Res. 22(10), 1557 (2007). https://doi.org/10.1359/ jbmr.070703
50. A. Snigirev, I. Snigireva, V. Kohn, S. Kuznetsov and I. Schelokov, "On the possibilities of $\mathrm{X}$-ray phase contrast microimaging by coherent high-energy synchrotron radiation", Rev. Sci. Instrum. 66(12), 5486 (1995). https://doi.org/10.1063/1.1146073

51. P. Weiss, L. Obadia, D. Magne, X. Bourges, C. Rau, T. Weitkamp, I. Khairoun, J.M. Bouler, D. Chappard, O. Gauthier and G. Daculsi, "Synchrotron X-ray microtomography (on a micron scale) provides three-dimensional imaging representation of bone ingrowth in calcium phosphate biomaterials", Biomaterials 24(25), 4591 (2003). https://doi. org/10.1016/S0142-9612(03)00335-1

52. D. Paganin, S.C. Mayo, T.E. Gureyev, P.R. Miller and S.W. Wilkins, "Simultaneous phase and amplitude extraction from a single defocused image of a homogeneous object", J. Microsc. 206(Pt 1), 33 (2002). https://doi.org/10.1046/j.1365-2818.2002.01010.x

53. U. Wolfram, J.J. Schwiedrzik, M.J. Mirzaali, A. Bürki, P. Varga, C. Olivier, F. Peyrin and P.K. Zysset, "Characterizing microcrack orientation distribution functions in osteonal bone samples", J. Microsc. 264(3), 268 (2016). https://doi.org/10.1111/ jmi.12440

54. M. Langer, A. Pacureanu, H. Suhonen, Q. Grimal, P. Cloetens and F. Peyrin, "X-ray phase nanotomography resolves the 3D human bone ultrastructure", PLoS One 7(8), e35691 (2012). https://doi. org/10.1371/journal.pone.0035691

55. M. Dierolf, A. Menzel, P. Thibault, P. Schneider, C.M. Kewish, R. Wepf, O. Bunk and F. Pfeiffer, "Ptychographic X-ray computed tomography at the nanoscale", Nature 467(7314), 436 (2010). https:// doi.org/10.1038/nature09419

56. N. Nango, S. Kubota, T. Hasegawa, W. Yashiro, A. Momose and K. Matsuo, "Osteocyte-directed bone demineralization along canaliculi", Bone 84, 279 (2016). https://doi.org/10.1016/j.bone.2015.12.006

57. G. Tawil, F. Jamme, M. Réfrégiers, A. ViksøNielsen, P. Colonna and A. Buléon, "In situ tracking of enzymatic breakdown of starch granules by synchrotron UV fluorescence microscopy", Anal. Chem. 83(3), 989 (2011). https://doi.org/10.1021/ ac1027512

58. E. Batard, F. Jamme, S. Villette, C. Jacqueline, M.-F. de la Cochetière, J. Caillon and M. Réfrégiers, "Diffusion of ofloxacin in the endocarditis vegetation assessed with synchrotron radiation UV 
fluorescence microspectroscopy", PLoS One 6(4), e19440 (2011). https://doi.org/10.1371/journal. pone. 0019440

59. G.A. Wagnières, W.M. Star and B.C. Wilson, "In vivo fluorescence spectroscopy and imaging for oncological applications", Photochem. Photobiol. 68(5), 603 (1998). https://doi. org/10.1111/j.1751-1097.1998.tb02521.x

60. N. Ramanujam, M.F. Mitchell, A. Mahadevan, S. Warren, S. Thomsen, E. Silva and R. RichardsKortum, "In vivo diagnosis of cervical intraepithelial neoplasia using 337-nm-excited laser-induced fluorescence", Proc. Natl. Acad. Sci. USA

91(21), 10193 (1994). https://doi.org/10.1073/ pnas.91.21.10193

61. G.Y. Rochefort, S. Pallu and C.L. Benhamou, "Osteocyte: the unrecognized side of bone tissue", Osteoporos. Int. 21(9), 1457 (2010). https://doi. org/10.1007/s00198-010-1194-5

62. S. Pallu, G.Y. Rochefort, C. Jaffre, M. Refregiers, D.B. Maurel, D. Benaitreau, E. Lespessailles, F. Jamme, C. Chappard and C.-L. Benhamou, "Synchrotron ultraviolet microspectroscopy on rat cortical bone: involvement of tyrosine and tryptophan in the osteocyte and its environment", PLoS One 7(8), e43930 (2012). https://doi.org/10.1371/journal. pone.0043930

63. L.M. Miller, J. Tibrewala and C.S. Carlson, "Examination of bone chemical composition in osteoporosis using fluorescence-assisted synchrotron infrared microspectroscopy", Cell. Mol. Biol. 46(6), 1035 (2000).

64. L.M. Miller, W. Little, A. Schirmer, F. Sheik, B. Busa and S. Judex, "Accretion of bone quantity and quality in the developing mouse skeleton", J. Bone Miner. Res. 22(7), 1037 (2007). https://doi.org/10.1359/ jbmr.070402

65. A.L. Boskey and R. Mendelsohn, "Infrared spectroscopic characterization of mineralized tissues", Vib. Spectrosc. 38(1-2), 107 (2005). https://doi. org/10.1016/j.vibspec.2005.02.015

66. A. Boskey and R. Mendelsohn, "Infrared analysis of bone in health and disease", J. Biomed. Opt. 10(3), 031102 (2005). https://doi.org/10.1117/1.1922927

67. S. Pallu, D.B. Maurel, C. Jaffré, R. Uzbekov, G.Y. Rochefort and P. Dumas, "Multi-modal imaging of osteocyte in an experimental model of alcohol-osteoporosis", presented at the Symposium JFFoE, Kyoto, Japan (2012).

68. R. Gauthier, M. Langer, H. Follet, C. Olivier, P.-J. Gouttenoire, L. Helfen, F. Rongiéras, D. Mitton and F. Peyrin, "3D micro structural analysis of human cortical bone in paired femoral diaphysis, femoral neck and radial diaphysis", J. Struct. Biol. 204(2), 182 (2018). https://doi.org/10.1016/j.jsb.2018.08.006

69. Y. Carter, C.D.L. Thomas, J.G. Clement and D.M.L. Cooper, "Femoral osteocyte lacunar density, volume and morphology in women across the lifespan", J. Struct. Biol. 183(3), 519 (2013). https://doi. org/10.1016/j.jsb.2013.07.004

70. Y. Carter, J.L. Suchorab, C.D.L. Thomas, J.G. Clement and D.M.L. Cooper, "Normal variation in cortical osteocyte lacunar parameters in healthy young males", J. Anat. 225(3), 328 (2014). https://doi. org/10.1111/joa.12213

71. Y. Carter, C.D.L. Thomas, J.G. Clement, A.G. Peele, K. Hannah and D.M.L. Cooper, "Variation in osteocyte lacunar morphology and density in the human femur-a synchrotron radiation micro-CT study", Bone 52(1), 126 (2013). https://doi. org/10.1016/j.bone.2012.09.010

72. K.M. Hannah, C.D.L. Thomas, J.G. Clement, F. De Carlo and A.G. Peele, "Bimodal distribution of osteocyte lacunar size in the human femoral cortex as revealed by micro-CT", Bone 47(5), 866 (2010). https://doi.org/10.1016/j.bone.2010.07.025

73. F.L. Bach-Gansmo, A. Brüel, M.V. Jensen, E.N. Ebbesen, H. Birkedal and J.S. Thomsen, "Osteocyte lacunar properties and cortical microstructure in human iliac crest as a function of age and sex", Bone 91, 11 (2016). https://doi.org/10.1016/j. bone.2016.07.003

74. P. Dong, S. Haupert, B. Hesse, M. Langer, P.-J. Gouttenoire, V. Bousson and F. Peyrin, "3D osteocyte lacunar morphometric properties and distributions in human femoral cortical bone using synchrotron radiation micro-CT images", Bone 60, 172 (2014). https://doi.org/10.1016/j. bone.2013.12.008

75. B. Hesse, P. Varga, M. Langer, A. Pacureanu, S. Schrof, N. Männicke, H. Suhonen, P. Maurer, P. Cloetens, F. Peyrin and K. Raum, "Canalicular network morphology is the major determinant of the spatial distribution of mass density in human bone tissue: evidence by means of synchrotron 
radiation phase-contrast nano-CT", J. Bone Miner. Res. 30(2), 346 (2015). https://doi.org/10.1002/ jbmr.2324

76. B. Hesse, N. Männicke, A. Pacureanu, P. Varga, M. Langer, P. Maurer, F. Peyrin and K. Raum, "Accessing osteocyte lacunar geometrical properties in human jaw bone on the submicron length scale using synchrotron radiation $\mu C C^{\prime \prime}$, J. Microsc. 255(3), 158 (2014). https://doi.org/10.1111/jmi.12147

77. B. Hesse, M. Langer, P. Varga, A. Pacureanu, P. Dong, S. Schrof, N. Männicke, H. Suhonen, C. Olivier, P. Maurer, G.J. Kazakia, K. Raum and F. Peyrin, "Alterations of mass density and 3D osteocyte lacunar properties in bisphosphonate-related osteonecrotic human jaw bone, a synchrotron $\mu \mathrm{CT}$ study", PLoS One 9(2), e88481 (2014). https://doi. org/10.1371/journal.pone.0088481

78. C. Albert, J. Jameson, P. Smith and G. Harris, "Reduced diaphyseal strength associated with high intracortical vascular porosity within long bones of children with osteogenesis imperfecta", Bone 66, 121 (2014). https://doi.org/10.1016/j. bone.2014.05.022

79. M.G. Mullender, S.D. Tan, L. Vico, C. Alexandre and J. Klein-Nulend, "Differences in osteocyte density and bone histomorphometry between men and women and between healthy and osteoporotic subjects", Calcif. Tissue Int. 77(5), 291 (2005). https://doi. org/10.1007/s00223-005-0043-6

80. B.R. McCreadie and S.J. Hollister, "Strain Concentrations surrounding an ellipsoid model of lacunae and osteocytes", Comput. Methods Biomech. Biomed. Engin. 1(1), 61 (1997). https://doi. org/10.1080/01495739708936695

81. N.K. Wittig, F.L. Bach-Gansmo, M.E. Birkbak, M. Laugesen, A. Brüel, J.S. Thomsen and H. Birkedal, "Organ and tissue level properties are more sensitive to age than osteocyte lacunar characteristics in rat cortical bone", Bone Rep. 4, 28 (2015). https://doi. org/10.1016/j.bonr.2015.11.003

82. H.M. Britz, Y. Carter, J. Jokihaara, O.V. Leppänen, T.L.N. Järvinen, G. Belev and D.M.L. Cooper, "Prolonged unloading in growing rats reduces cortical osteocyte lacunar density and volume in the distal tibia", Bone 51(5), 913 (2012). https://doi. org/10.1016/j.bone.2012.08.112

83. S.M. Tommasini, A. Trinward, A.S. Acerbo, F. De Carlo, L.M. Miller and S. Judex, "Changes in intracortical microporosities induced by pharmaceutical treatment of osteoporosis as detected by high resolution micro-CT", Bone 50(3), 596 (2012). https://doi.org/10.1016/j. bone.2011.12.012

84. K. Matsuo, Y. Kuroda, N. Nango, K. Shimoda, Y. Kubota, M. Ema, L. Bakiri, E.F. Wagner, Y. Takeda, W. Yashiro and A. Momose, "Osteogenic capillaries orchestrate growth plate-independent ossification of the malleus", Development 142(22), 3912 (2015). https://doi.org/10.1242/dev.123885

85. J.A. Núñez, A. Goring, B. Javaheri, H. Razi, D. Gomez-Nicola, A.A. Pitsillides, P.J. Thurner, D. Gomez-Nicola, P. Schneider and C.E. Clarkin, "Regional diversity in the murine cortical vascular network is revealed by synchrotron X-ray tomography and is amplified with age", Eur. Cell. Mater. 35, 281 (2018). https://doi.org/10.22203/eCM.v035a20

86. A. Carriero, M. Doube, M. Vogt, B. Busse, J. Zustin, A. Levchuk, P. Schneider, R. Müller and S.J. Shefelbine, "Altered lacunar and vascular porosity in osteogenesis imperfecta mouse bone as revealed by synchrotron tomography contributes to bone fragility", Bone 61, 116 (2014). https://doi.org/10.1016/j. bone.2013.12.020

87. A. Karunaratne, L. Xi, L. Bentley, D. Sykes, A. Boyde, C.T. Esapa, N.J. Terrill, S.D.M. Brown, R.D. Cox, R.V. Thakker and H.S. Gupta, "Multiscale alterations in bone matrix quality increased fragility in steroid induced osteoporosis", Bone 84, 15 (2016). https:// doi.org/10.1016/j.bone.2015.11.019

88. H. Mosey, J.A. Núñez, A. Goring, C.E. Clarkin, K.A. Staines, P.D. Lee, A.A. Pitsillides and B. Javaheri, "Sost deficiency does not alter bone's lacunar or vascular porosity in mice", Front. Mater. 4, 27 (2017). https://doi.org/10.3389/fmats.2017.00027

89. K.S. Mader, P. Schneider, R. Müller and M. Stampanoni, "A quantitative framework for the 3D characterization of the osteocyte lacunar system", Bone 57(1), 142 (2013). https://doi.org/10.1016/j. bone.2013.06.026

90. M. Gerbaix, V. Gnyubkin, D. Farlay, C. Olivier, P. Ammann, G. Courbon, N. Laroche, R. Genthial, H. Follet, F. Peyrin, B. Shenkman, G. Gauquelin-Koch and L. Vico, "One-month spaceflight compromises the bone microstructure, tissue-level mechanical properties, osteocyte survival and lacunae volume 
in mature mice skeletons", Sci. Rep. 7(1), 2659 (2017). https://doi.org/10.1038/s41598-017-03014-2

91. M. Kerschnitzki, P. Kollmannsberger, M. Burghammer, G.N. Duda, R. Weinkamer, W. Wagermaier and P. Fratzl, "Architecture of the osteocyte network correlates with bone material quality", J. Bone Miner. Res. 28(8), 1837 (2013). https://doi.org/10.1002/ jbmr.1927

92. A. Ciani, M. Guizar-Sicairos, A. Diaz, M. Holler, S. Pallu, Z. Achiou, R. Jennane, H. Toumi, E. Lespessaille and C.M. Kewish, "Segmentation of nanotomographic cortical bone images for quantitative characterization of the osteoctyte lacuno-canalicular network", AIP Conf. Proc. 1696, 020040 (2016). https://doi.org/10.1063/1.4937534

93. A. Ciani, H. Toumi, S. Pallu, E.H.R. Tsai, A. Diaz, M. Guizar-Sicairos, M. Holler, E. Lespessailles and C.M. Kewish, "Ptychographic X-ray CT characterization of the osteocyte lacuno-canalicular network in a male rat's glucocorticoid induced osteoporosis model", Bone Rep. 9, 122 (2018). https://doi.org/10.1016/j. bonr.2018.07.005

94. F. Jamme, S. Kascakova, S. Villette, F. Allouche, S. Pallu, V. Rouam and M. Réfrégiers, "Deep UV autofluorescence microscopy for cell biology and tissue histology", Biol. Cell 105(7), 277 (2013). https://doi.org/10.1111/boc.201200075

95. R.K. Fuchs, M.R. Allen, M.E. Ruppel, T. Diab, R.J. Phipps, L.M. Miller and D.B. Burr, "In situ examination of the time-course for secondary mineralization of Haversian bone using synchrotron
Fourier transform infrared microspectroscopy", Matrix Biol. 27(1), 34 (2008). https://doi. org/10.1016/j.matbio.2007.07.006

96. R.Y. Huang, L.M. Miller, C.S. Carlson and M.R. Chance, "In situ chemistry of osteoporosis revealed by synchrotron infrared microspectroscopy", Bone 33(4), 514 (2003). https://doi.org/10.1016/S87563282(03)00233-3

97. G.J. Kazakia, D. Speer, S. Shanbhag, S. Majumdar, B.R. Conklin, R.A. Nissenson and E.C. Hsiao, "Mineral composition is altered by osteoblast expression of an engineered G(s)-coupled receptor", Calcif. Tissue Int. 89(1), 10 (2011). https://doi. org/10.1007/s00223-011-9487-z

98. M. Holler, A. Diaz, M. Guizar-Sicairos, P. Karvinen, E. Färm, E. Härkönen, M. Ritala, A. Menzel, J. Raabe and O. Bunk, "X-ray ptychographic computed tomography at $16 \mathrm{~nm}$ isotropic 3D resolution", Sci. Rep. 4(1), 3857 (2014). https://doi.org/10.1038/ srep03857

99. S. Hirose, M. Li, T. Kojima, P.H.L. de Freitas, S. Ubaidus, K. Oda, C. Saito and N. Amizuka, "A histological assessment on the distribution of the osteocytic lacunar canalicular system using silver staining", J. Bone Miner. Metab. 25(6), 374 (2007). https://doi.org/10.1007/s00774-007-0764-x

100. M. Ferretti, M.A. Muglia, F. Remaggi, V. Canè and C. Palumbo, "Histomorphometric study on the osteocyte lacuno-canalicular network in animals of different species. II. Parallel-fibered and lamellar bones", Ital. J. Anat. Embryol. 104(3), 121 (1999). 